Henrike Helmer*

\title{
Analepsen mit Topik-Drop. Zur Notwendigkeit einer diskurssemantischen Perspektive
}

\section{Analepses with Topic-Drop. On the Necessity of a Discourse Semantic Perspective}

DOI 10.1515/zgl-2017-0001

Abstract: Analepses with topic-drop are frequent structures in German interaction. While hitherto the focus on analepses was a rather syntactic one, this paper deals with analeptic structures from a semantic perspective. It particularly concentrates on the semantic relations between the referents of the analepses and the prior interactional context. This analysis shows that even for rather simple analepses which just omit a constituent from the prior utterance, conceptual processes are more decisive for its interpretation than syntactic features of the antecedent constituents. This is even more the case for complex analepses that are only indirectly linked to the prior context, and for the interpretation of which hearers need to draw inferences. The paper argues that theoretical approaches like Conversation Analysis and Interactional Linguistics can profit from adopting a semantic and conceptual perspective for the interpretation of interactional structures.

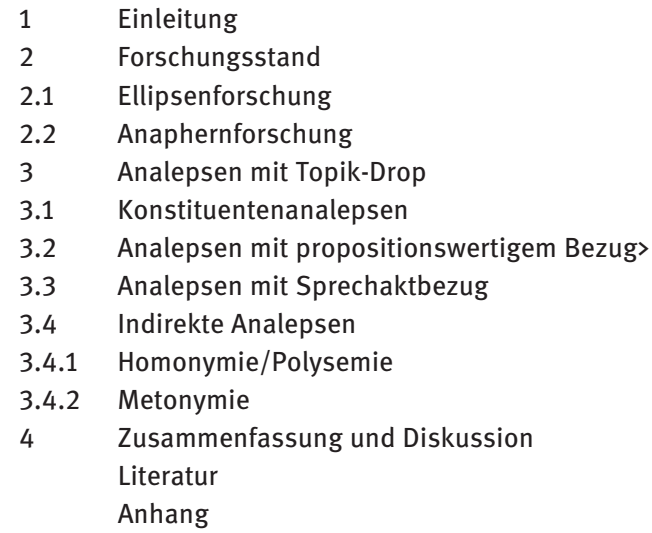

*Kontaktperson: Dr. Henrike Helmer: Institut für Deutsche Sprache, R5, 6-13, D-68161 Mannheim, E-Mail: helmer@ids-mannheim.de 


\section{Einleitung}

Analepsen mit Topik-Drop sind hochfrequente sprachliche Strukturen im gesprochenen Deutsch. Als Analepsen werden satzgrammatisch unvollständige Äußerungen bezeichnet, die auf „die „Kontinuität des Redens“ bezogen sind (Hoffmann 1999: 72), und in denen das Topik einer vorherigen Äußerung nicht erneut verbalisiert wird. Das Topik ist das, was kontextuell bekannt ist und über das etwas ausgesagt wird (vgl. z. B. Reinhart 1991, Lambrecht 1994, sowie als Überblick Musan 2002, Krifka/Musan 2012), in diesem Fall durch die analeptische Äußerung. ${ }^{1}$

In diesem Beitrag stehen diskurssemantische Perspektiven und Fragestellungen im Vordergrund, insbesondere die detaillierte Beschreibung der semantischen Relationen zwischen Analepsen und ihrem Präkontext. Die Interpretation von Analepsen muss situiert erklärt werden, da sie von der kontextuellen Einbettung sowie von grammatischen, semantischen und pragmatischen Merkmalen der Äußerung abhängt.

Durch das Nichtexplizieren des Topiks können Sprecher den Aufwand bei der Produktion von Analepsen gering halten, wenn sie davon ausgehen können, dass die Hörer den Bezug der Analepse auf der Basis ihres Diskurs-, Situationsund Weltwissens ohne Probleme nachvollziehen können. Der höhere Aufwand beim Verarbeitungsprozess für den Hörer ist dabei i. d. R. kein Problem, z. T. bietet die Indetermination diesem sogar rhetorische Vorteile für Anschlussäußerungen. Auf die sprechökonomischen, informationsstrukturellen und rhetorischen Funktionen von Analepsen werde ich im Folgenden jedoch nur am Rande eingehen. Stattdessen liegt der Fokus auf der Frage, ob Sprecher von Analepsen Elemente vorangehender Äußerungen als syntaktische Strukturen nutzen, oder ob eher konzeptuelle Vorgänge und Repräsentationen der Elemente des Präkontexts ausschlaggebend sind.

In der bisherigen Forschung wurden vor allem Analepsen besprochen, in denen einzelne Konstituenten nicht realisiert werden. Im diesem Artikel dagegen werden u. a. spezifische Analepsentypen diskutiert, die etwa von Auer (1993) als Fälle von ,uneigentlicher Verbspitzenstellung ${ }^{2}{ }^{2}$ untersucht worden sind. Dies sind sprachliche Strukturen, bei denen „das Verb deshalb am Anfang [steht],

1 Insbesondere da dies äußerungsübergreifend passiert, wird im Folgenden von ,Topik‘ gesprochen und nicht etwa der Begriff ,Thema‘ verwendet, der traditionellerweise eher satzorientiert verwendet wird (aber nicht nur, vgl. etwa Zifonun et al. (1997: 508ff.)).

2 In meiner Kollektion handelt es sich allerdings nicht immer um Verbspitzenstellung, bei einigen der untersuchten Analepsen findet sich auch die Verbzweitstellung. 
weil dem Satz eine seiner obligatorischen Ergänzungen fehlt“ (Auer 1993: 198). Dabei ist die gesamte Vorgängeräußerung das Topik, das in der Analepse nicht erneut realisiert wird und über das etwas ausgesagt wird. Diese Art von Analepsen können unter den Begriff der Vorwärtsellipse (Klein 1993: 770) in einem weiten Verständnis gefasst werden (sofern man unter diesen Begriff auch nicht koordinierte Ellipsen subsumiert, da es sich nicht um Koordinationsellipsen handelt, s. Abschnitt 2.1). Die Forschungen zur ,uneigentlichen Verbspitzenstellung' thematisieren jedoch expliziter die im Folgenden besprochenen Strukturen.

Zum einen werde ich diskutieren, ob im Falle von Konstituentenanalepsen von syntaktischen oder phonologischen Strukturübernahmen ausgegangen werden kann, zum anderen werde ich Analepsen präsentieren, bei denen solche Strukturübernahmen eindeutig nicht möglich sind: nämlich Analepsen mit ausgelassenem propositionswertigem Topik, sowie indirekte Analepsen, deren ausgelassenes Topik gar nicht direkt im Präkontext geäußert wurde, sondern nur indirekt mit zuvor geäußerten sprachlichen Strukturen zusammenhängt. Im Gegensatz zu Konstituentenanalepsen, bei denen das Topik i. d. R. leicht zu explizieren ist, handelt es sich bei den untersuchten Analepsen häufig um Typen von Topik-Auslassungen, für die eine verbale Explizierung für Sprecher nicht nur einfach unökonomisch wäre, sondern bisweilen sogar kaum sinnvoll auf den Punkt zu bringen wäre.

Bei der empirischen Untersuchung analeptischer Äußerungen mit diskurssemantischem Schwerpunkt wird ersichtlich, dass Analepsen häufig komplexer sind, als i.d. R. angenommen wird, wenn der Fokus auf der Analyse von Konstituentenanalepsen liegt. Daher stellt sich die Frage, aufgrund welcher konzeptuellen Vorgänge Analepsen überhaupt geäußert und verstanden werden können. Damit einher gehen Konsequenzen für traditionell eher agnostisch ausgerichtete Ansätze wie die Konversationsanalyse und Interaktionale Linguistik, in denen bisher sowohl semantische Analysen größtenteils vernachlässigt wurden als auch Annahmen über Kognitionen der Interaktionsbeteiligten als zu spekulativ abgelehnt wurden (Gegenbeispiele aus der Interaktionalen Linguistik sind aber z. B. Imo 2011, Deppermann 2012).

Nach einem Forschungsüberblick zu Ellipsen, Analepsen und Anaphern (Abschnitt 2) werde ich zunächst Analepsen vorstellen, die wie Konstituentenanalepsen wirken, aber nicht mit einer direkten Übernahme der Antezedenzien zu erklären sind (Abschnitt 3.1). Danach werde ich komplexere Analepsentypen diskutieren, nämlich Analepsen mit nicht realisiertem propositionswertigem Topik (Abschnitt 3.2), Analepsen mit Sprechaktbezug (Abschnitt 3.3) und indirekte Analepsen (Abschnitt 3.4), deren Topik nur indirekt mit Elementen des Präkontextes zusammenhängt und für deren Produktion und Interpretation daher kom- 
plexere Inferenzen nötig sind. Abschließend werde ich diskutieren, inwiefern die Untersuchung speziell indirekter Analepsen sowohl für die Ellipsenforschung als auch für die Konversationsanalyse von Bedeutung ist (Abschnitt 4).

\section{Forschungsstand}

Da in der Ellipsenforschung (Abschnitt 2.1) tendenziell ein syntaktischer Fokus bei der Beschäftigung mit Ellipsen und Analepsen zu beobachten ist, erscheint eine Kombination mit Erkenntnissen und Konzepten der Anaphernforschung (Abschnitt 2.2) ertragreich, um einen eher semantischen Blick auf analeptische Strukturen zu gewinnen.

\subsection{Ellipsenforschung}

In der Tradition der Ellipsenforschung gibt es Auseinandersetzungen zu verschiedenen Aspekten, von denen der bekannte Dissenz zwischen „Ableitern“ (Begriff nach Selting 1997) und „Autonomisten“ nur einer ist, d. h. der Disput, ob Ellipsen als reduzierte Formen vollständiger Sätze (z. B. Klein 1993, Helbig/ Buscha 1999, Eroms 2000, Engel 2009) oder als eigenständige sprachliche Muster zu begreifen sind, aus denen sich kein vollständiger Satz rekonstruieren lässt (z. B. Kindt 1985, Busler/Schlobinski 1997, Zifonun et al. 1997, Hoffmann 1999; sowie viele Autoren aus dem Bereich der Gesprochene-Sprache-Forschung: Selting 1997, Lindström/Karlsson 2005, Auer 2007, 2014, Deppermann 2006, 2007, Günthner 2006, Imo 2011, 2013, 2016, Bücker 2012). So existieren neben diesen theoretischen Uneinigkeiten unterschiedliche Terminologien (etwa Rath 1979, Klein 1993, Zifonun et al. 1997, Hoffmann 1997, 1999, Busler/ Schlobinski 1997, Auer 2007, 2014, 2015), unterschiedlich groß definierte Umfänge des Phänomenbereichs (vgl. etwa Selting 1997 und Busler und Schlobinski 1997) und Unstimmigkeiten hinsichtlich der Frage, ob Ellipsen und Vollständigkeit unter syntaktischen oder semantischen Gesichtspunkten verstanden werden muss (vgl. etwa Hoffmann 1999 und Auer 2014, 2015). Einig sind sich i. d. R. alle Autoren, dass Äußerungen, die zwar syntaktisch unvollständig sind, durchaus trotzdem kommunikativ vollständig sein können, also z. B. eine eigenständige Sprechhandlung darstellen können. Zudem scheint seit einiger Zeit größtenteils Einigkeit darüber zu bestehen, dass die Arbeit mit empirischen Daten dem Erfinden von Beispielen vorzuziehen ist (vgl. etwa Hennig 2013).

In die Klasse der syntaktisch unvollständigen Strukturen (die allerdings kommunikativ vollständig sind) lassen sich unterschiedliche Strukturen ein- 
ordnen, die traditionell als ,elliptisch' bezeichnet werden. Nicht behandelt werden im Folgenden idiomatische bzw. grammatikalisierte elliptische Strukturen, sowie Ellipsen die auf „gemeinsamer Orientierung in der Sprechsituation, im aktuellen Handlungszusammenhang oder auf Basis sprachlichen Wissens“ (Zifonun et al. 1997: 413) beruhen und daher ohne den lokalen sequenziellen Kontext verstanden werden können, etwa situative Ellipsen, Ereignis-Ellipsen, Objekt-Ellipsen, empraktische Ellipsen und phatische Ellipsen (vgl. Zifonun et al. 1997: 409ff.). Stattdessen konzentriere ich mich im Folgenden auf die von Hoffmann $(1997,1999)$ funktional(grammatisch) diskutierten Analepsen. Diese beruhen ,auf vorgängiger Verbalisierung, die unter bestimmten Bedingungen in Geltung bleibt, so daß das, was folgt, unmittelbar angeschlossen bzw. koordinativ eingebunden werden kann“ (Hoffmann 1999: 72). Objektellipsen, Ereignisellipsen u. Ä. sind daher u. U. kommunikativ „restringierter“ (die gemeinsame Wahrnehmung muss gewährleistet sein), bei Analepsen kann fast alles Verbalisierte im Präkontext als Antezedens dienen, womit andere Erfordernisse und Restriktionen, aber auch andere Möglichkeiten der Bezugnahme, für die Interpretation sowie weitere funktionale Eigenschaften einhergehen (dazu ausführlicher Helmer 2016). Im Folgenden wird der Fokus nicht auf Analepsen gelegt, die auf Koordination beruhen (wie beim gapping und sluicing, vgl. Carnie 2013: 457ff.), sondern auf Adjanzenzanalepsen, die zudem selbst ein Verb enthalten. Eine Typologie von ,unvollständigen Äußerungen “ und eine Abgrenzung des Untersuchungsgegenstands ist Abbildung 1 zu entnehmen. 


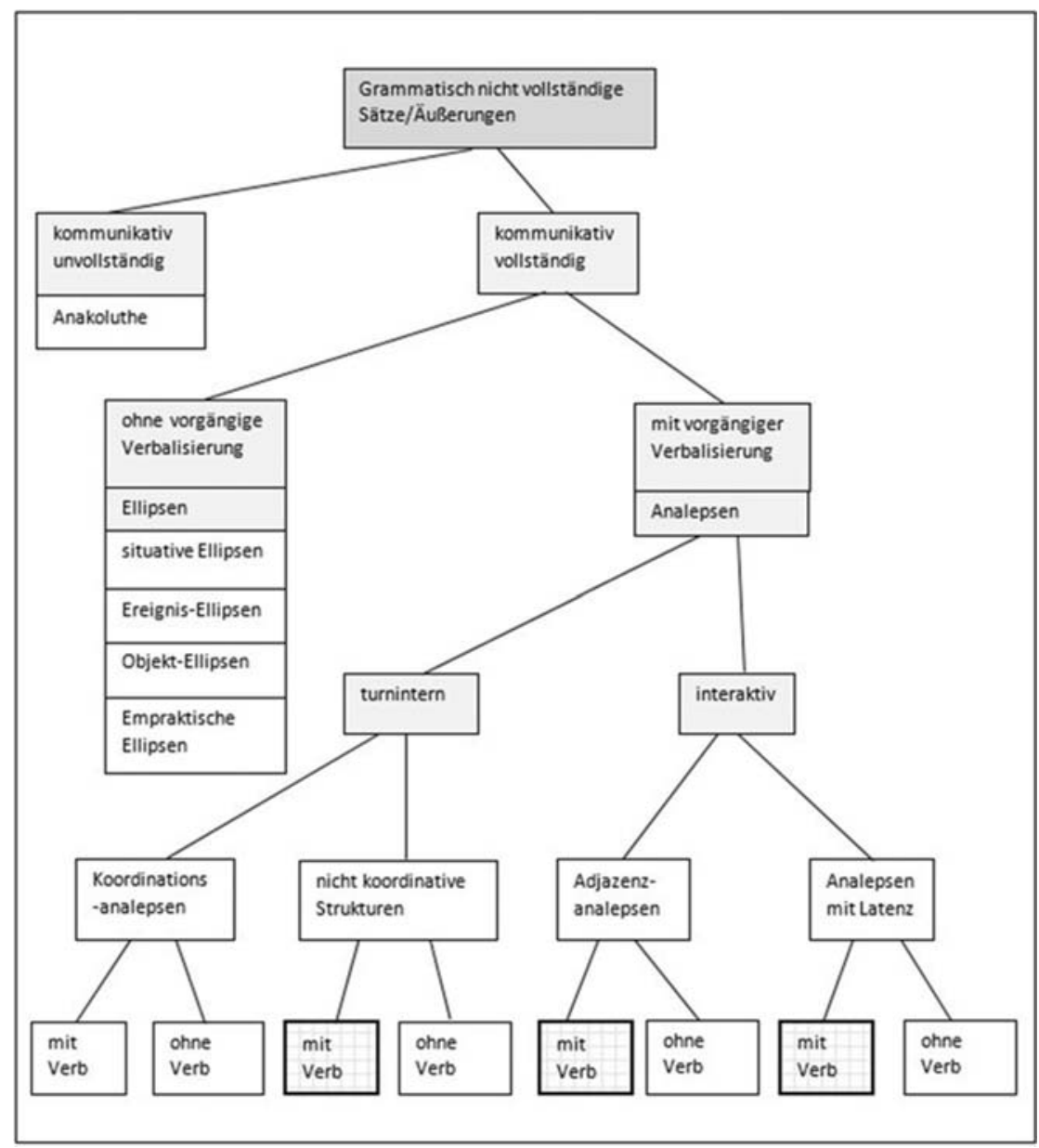

Abb. 1: Typologie syntaktisch nicht vollständiger Äußerungen und Abgrenzung des Untersuchungsgegenstands (dick umrandet)

Bereits Rath (1979), Kindt (1985) und Ortner (1987) betonen die Bedeutung der Sequenzialität für Analepsen. Die von Rath (1979) untersuchten ,Konstruktionsübernahmen' analysiert Auer (2007, 2014, 2015) in natürlich-sequenzieller verbaler Interaktion detailliert als ,Strukturlatenzen“. Er betont den linearen, dialogorientierten und dabei inkrementellen Charakter gesprochener Sprache (Auer 2000, 2006, 2007) und die „maximale Synchronisiertheit von Produktion und Rezeption“ (Auer 2007: 96). Er kritisiert und widerlegt die verbreitete Annahme, eine syntaktische Struktur bestehe nur ko-extensiv mit der Zeit der Produktion und Rezeption (Auer 2014). Er ist demgegenüber der Ansicht, dass sprachliche Äußerungen struk- 
turelle Latenzen bereitstellen, die auch später wiederverwendet werden können. Als Illustration wählt Auer die biologische Metapher der ,unilateralen Symbiose‘: Ein symbiont koppelt sich an einen Gastgeber, host, ohne den er nicht leben kann, während der host seinerseits ohne den symbiont leben kann. Auf linguistischer Ebene kopiert analog eine analeptische Folgeäußerung (symbiont) eine Struktur der Vorgängeräußerung (host), ohne die sie nicht bestehen kann (Auer 2014: 535). Gemeint ist dabei nicht, dass der symbiont-Äußerung eine Tiefenstruktur zugrunde liegt, sondern dass die zu einer Vollform „fehlenden“ Teile durch die host-Äußerung noch zugänglich sind. Diese Zugänglichkeit beginnt dabei gewissermaßen koextensiv mit der Artikulation der Strukturlatenzen: „The temporal alignment of participants in dialogue starts already at that point: with a shared array of latent structures on which next speakers (and listeners) can build“ (Auer 2015: 48).

Auers Ansatz stellt ein für viele Fälle von Analepsen aufschlussreiches Modell bereit. Auer (2014) diskutiert bis auf einen Fall von Pro-Drop aber ausschließlich verblose Analepsen. Eine nähere Betrachtung der Darstellungsweise von Auer (2014) lässt hingegen vermuten, dass eine hinreichende Interpretation von Analepsen nicht immer allein auf der Basis des lokalen syntaktischen Kontextes funktionieren kann, sondern nur dann, wenn das Verb einer vorhergehenden Äußerung die Strukturlatenzen herstellt und die Analepse „mitregiert“. Auch Plewnia (2003: 42ff.) grenzt Ellipsen mit syntaktischem Kontextbezug von solchen mit semantischen Bezugnahmen ab (s. u.). Für Analepsen, die selbst ein Verb enthalten, erscheint letzteres sinnvoller. Exemplarisch sei in Abbildung 2 ein Beispiel von Auer gezeigt: ${ }^{3}$

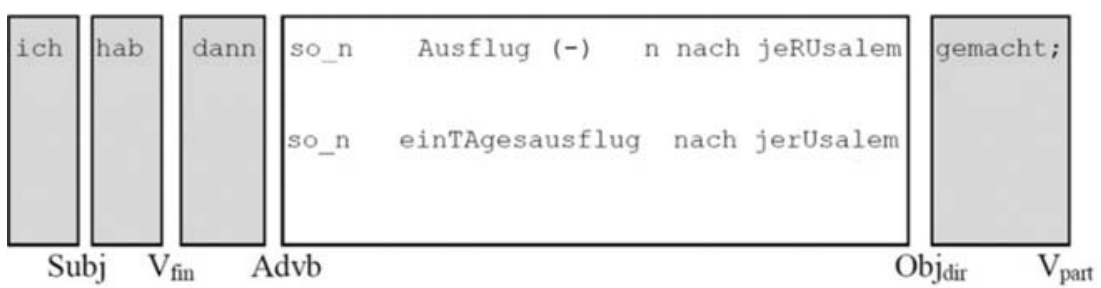

Abb. 2: Die Strukturübernahme bei einer analeptischen Äußerung (nach Auer 2014: 541)

3 Eine ähnliche Darstellungsweise wählt Du Bois für die „dialogic syntax“ (Du Bois 2014) bzw. Resonanzstrukturen, die er beobachtet, wenn Sprecher aufeinander bezogene Turns mit syntaktischen und semantischen Parallelen formulieren. Allerdings betrachtet Du Bois nicht explizit analeptische Äußerungen (auch wenn sie in seinen Analysen vorkommen). 
Dies ist eine sehr geeignete Darstellung von Strukturen mit elidiertem Verbalkomplex, die Expansionen, Reparaturen, kollaborative Äußerungen u. Ä. sind. Bei Analepsen, die selbst ein Verb enthalten, ist eine Darstellung wie in Abbildung 2 dagegen i. d. R. problematisch. Zum einen wechselt oftmals der Gegenstand, über den gesprochen wird, seinen syntaktischen Status, je nachdem, ob man vom Antezedens als Argument der Vorgängeräußerung ausgeht oder vom nicht-realisierten Argument des Verbs der Analepse (das im Falle der Realisierung und im Falle von Koreferenz aber auf denselben Gegenstand Bezug nehmen würde wie das Antezedens). Zum anderen sind analeptische Äußerungen, in denen ein Argument eines realisierten Verbs weggelassen wird, häufig neue Sprechhandlungen wie Bewertungen etc. Eine typische analeptische Äußerung dieser Art wäre war sehr schön als Bewertung des Ausflugs. Dieser wird im Antezedens als direktes Objekt realisiert (,so=n ausflug (-) n nach jeRUsalem“). In einer bewertenden Analepse wie war sehr schön würde jedoch das Subjekt fehlen. Die bei einer potenziellen Vollrealisierung der Analepse (etwa als Anapher) zu beobachtende grammatische Veränderung der Konstituente vom direkten Objekt (der Bezugsäußerung) zum Subjekt (der Analepse) würde eine solche Darstellung gewissermaßen unterschlagen (vgl. Abb. 3).

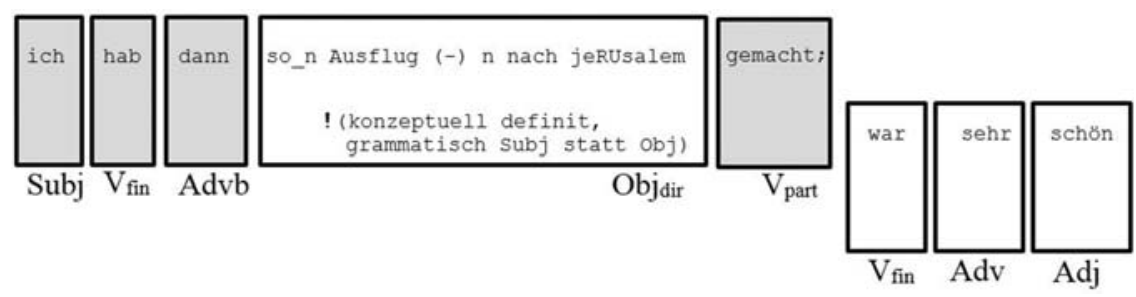

Abb. 3: Antezedens und Analepse bei fiktivem Beispiel, basierend auf Auer (2014: 541)

Wie man bei einem Vergleich der Abbildungen 2 und 3 sieht, wirken Bezugsäußerungen mit Verb für Analepsen ohne Verb prospektiv (das Verb der Bezugsäußerung kann spätere Konstituenten „mitregieren“); der Fokus bei der Interpretation von Analepsen, die selbst ein Verb enthalten, liegt dagegen eher auf der Retrospektivität (von der Analepse ausgehend muss im vorhergehenden Kontext das nicht realisierte Argument identifiziert werden).

Die Interpretation solcher Äußerungen vollzieht sich auf der konzeptuellen Ebene. Insbesondere Analepsen mit propositionalem Bezug sind zwar kontextkontrolliert (nach der Terminologie Kleins 1993), beruhen aber nicht auf Koordination 
und fallen deshalb nicht mehr unter den Begriff der Koordinationsellipse. ${ }^{4}$ Analepsen dieser Art sind sprachliche Strukturen, die Plewnia (2003: 56ff.) als Ellipsen mit semantischem Kontextbezug bzw. ,Externe Prädikationen' (nach Behr/Quentin (1996: 56-60) beschreibt. Solche Ellipsen operieren elementbezogen oder global „prädizierend von außen auf den Vorgängersatz“ (Plewnia 2003: 57). Auch Kindt konstatiert zu externen Prädikationen, dass es sich nicht um ,,,versteckte“ asyndetische Koordinationsellipse[n]“" handelt (Kindt 2013: 82). Ein rein syntaktischer Fokus, v. a. auf eine koordinative Eingebundenheit, erscheint für die Beschreibung und Erklärung von solchen Analepsen nicht angemessen.

Während bei einem syntaktischen Fokus konzeptuell interessante Vorgänge mitunter unterschlagen werden können, wird in anderen Ansätzen wie etwa in der Psycholinguistik (z. B. Rickheit/Sichelschmidt 2013) und z. T. der funktionalen Grammatik Unvollständigkeit bisweilen mit der Indexikalität von Sprache gleichgesetzt. So erklärt z. B. Hoffmann (1999: 70), ein Wirklichkeitsausschnitt könne nie exhaustiv sprachlich abgebildet werden, wodurch der Ansatz, zu fragen, was weggelassen werden könne, falsch sei (Hoffmann 1999: 71). Analepsen und Ellipsen sind aber auch ein grammatisches Phänomen. Zwar können kognitive Strukturen an sich immer inkrementell erweitert werden und Sprecher sagen i. d. R. gerade das, was Hörer für die hinreichende Verarbeitung der Äußerung benötigen; letztlich heben sich Ellipsen und Analepsen aber dadurch von anderen Äußerungen ab, die in irgendeiner Art semantisch/konzeptuell unterspezifiziert sind (wie etwa durch pronominale Anaphern), dass in ihnen nach valenzgrammatischen Vorstellungen bestimmte Verbergänzungen nicht realisiert sind.

Von Interesse ist also einerseits zu zeigen, wie Sprecher Analepsen zwar situativ und kommunikativ kontextgebunden äußern und verstehen (vgl. auch Rickheit/Strohner 2008), und dabei andererseits aufzuweisen, dass die kontextbasierte Interpretation nicht in einer direkten syntaktischen Übernahme aus dem sprachlichen Präkontext besteht.

4 Klein selbst unterscheidet zwischen Koordinationsellipsen (,innerhalb eines Satzes durch Koordination“, vgl. Klein 1993: 768) und Adjazenzellipsen (,kontrollierender Ausdruck und elliptischer [bilden] zwei selbstständige aber zusammengehörige Äußerungen“ (ebd.)), zwischen denen es zwar Schnittmengen geben kann (wie beim gapping), die v. a. bezüglich ihrer interaktiven Struktur jedoch auch Unterschiede aufweisen. 


\subsection{Anaphernforschung}

Ein mit Analepsen hinsichtlich der Bezugnahme auf den Präkontext verwandtes Phänomen ist die Bezugnahme durch Anaphern. Diese „sind Ausdrücke, die innerhalb eines Textes einen anderen Ausdruck (das Antezedens oder der Antezedent) wieder aufgreifen und mit denen ein Sprecher somit auf einen im Text oder Diskurs bereits erwähnten Referenten erneut Bezug nimmt“ (Consten/Schwarz-Friesel 2007: 265-266). Während in der Ellipsenforschung bisweilen implizit oder explizit die Vorstellung anklingt, Antezedenzien könnten in Analepsen „eingefügt“ werden bzw. für deren Produktion und Interpretation „kopiert“ werden, wurden in der eher textlinguistisch und empirisch orientierten Tradition der Anaphernforschung detailliert Phänomene untersucht, die mit Kopiervorstellungen nicht zu erklären sind, etwa Kasus- und Numerusinkongruenzen zwischen Antezedens und Anapher. ${ }^{5}$ Ebenfalls ist es für das Verständnis von Anaphern unproblematisch, wenn sich das Genus der Anapher nach dem Sexus des Referenten richtet, nicht nach dem Genus des syntaktischen Antezedens. ${ }^{6}$ Darüber hinaus sind Anaphern frequent, die sich auf satzwertige Antezedenten beziehen und die dort ausgedrückten Sachverhalte zu einem einheitlichen Diskursobjekt zusammenfassen (Consten/Marx 2006: 375). Diese werden von Consten/Marx (2006) und Consten/Schwarz-Friesel (2007) ,Komplexanaphern‘ genannt. In Texten finden sich nicht nur Demonstrativa als Komplexanaphern, sondern auch abstrakte definite Nominalphrasen wie diese Tatsache. Die Möglichkeit von Inkongruenzen sowie von Anaphern mit komplexeren Bezügen zeigt, dass nicht grammatische Merkmale für die Produktion oder das Verständnis von Anaphern konstitutiv sind, sondern in einigen Fällen vielmehr konzeptuelles Wissen und Koreferenz zwischen Antezedens und Anapher ausschlaggebend sind, in anderen Fällen Inferenzen. Bei Analepsen verhält es sich ähnlich: Auch wenn etwa das Verb der Analepse im Singular steht, kann das Antezedens bzw. der sprachliche Anker in einer vorherigen Äußerung eine Nominalphrase im Plural sein (vgl. z. B. Abschnitt 3.4.2).

\footnotetext{
5 Ein Beispiel für Numerusinkongruenz geben Consten und Schwarz-Friesel (2007: 274): „Für die Zeit [der Olympischen Spiele $]_{j}$ nehme ich mir nichts anderes vor, denn ich freue mich riesig auf [das Ereignis] ]. “ Unter dem hier weit gefassten Begriff der Numerusinkongruenz verstehen die Autoren den Wechsel vom Plural („die Olympischen Spiele“) zum Singular („das Ereignis“) bei gleichzeitiger Koreferenz. Dieser Begriff von Anapher ist allerdings ein recht weiter im Vergleich zum traditionellen Verständnis von ,Anapher“ als „Personalpronomen in der 3. Person“. (vgl. Zifonun et al. 1997: 544ff.).

6 Ein Beispiel hierfür ist nach (Consten/Schwarz-Friesel 2007: 274): „Ein Mädchen ${ }_{i}{\text { stieg ein. } \text { Sie }_{i}}$ sah sich hilflos um, weil sie ${ }_{i}$ keinen freien Platz fand.“
} 
In der Tradition der Anaphernforschung wurden nicht nur Anaphern untersucht, die Koreferenz zum Antezedens aufweisen. Forscher haben sich auch detailliert mit Anaphern ohne direkte Koreferenzrelation beschäftigt. Solche Anaphern sind im vorhergehenden Text nur indirekt verankert (Consten/Schwarz-Friesel 2007: 271), sodass der weiter zurückreichende sprachliche Kontext, Situations- oder Weltwissen und mit diesen zusammenhängende Inferenzen herangezogen werden müssen, um einen Referenten zu identifizieren. Solche Anaphern werden unterschiedlich bezeichnet. Clark (1975) erfasst die mentalen Prozesse bei der Interpretation mit dem Begriff bridging (assumptions). Während laut Clark direkte (Ko-) Referenz etwa durch Pronominalisierung oder Epitheta hergestellt wird (Clark 1975: 170), funktioniert indirekte Referenzherstellung durch Assoziationen, Implikaturen und Grund-, Ursprungs- oder Folgeverhältnisse zwischen Antezedens und der sich auf das Antezedens beziehenden Konstituente. Bezeichnungen von nicht koreferenziellen lexikalischen Anaphern sind ,Kontiguitäts-Anaphora“ (Harweg 1968), ,assoziative Anaphora' (Hawkins 1978), ,antezedenslose Rückverweise‘ (Kubczak 2012) oder der im Folgenden übernommene Begriff, indirekte Anaphern` (Consten/Schwarz-Friesel 2007). Die semantischen Relationen zwischen indirekten Anaphern und ihren „Ankern“7 (Schwarz 1997: 449) sind häufig metonymisch oder antonymisch oder es handelt sich um Bezüge auf das kumulative Diskurstopik. In jedem Fall besteht eine systematische Verbindung zweier unterschiedlicher konzeptueller Domänen. Der sprachliche Anker ist dabei gewissermaßen „Quellbereich“ für das Verständnis des „Zielbereichs“8 (Lakoff/Johnson 1980) der indirekten Anapher. Indirekte Anaphern sind in verschiedener Weise im Prätext verankert (vgl. Schwarz 2000). Sprachliche Anker der Anaphern können etwa gängiges (Welt-)Wissen im Langzeitgedächtnis aktivieren (über semantische Rollen oder Meronymien) oder bieten die Basis für Inferenzen, die ein Rezipient mithilfe semantischen und konzeptuellen Wissens ziehen muss.

Auch Computerlinguisten betonen die Relevanz der semantischen Ebene für geeignete Algorithmen zur Identifizierung von Antezedenzien von Anaphern, Identifizierung von Metonymien und Interpretation von bridging (z. B. Mitkov 1999, Markert 1999, Vieria/Poesio 2000, Markert/Hahn 2002, Markert/Nissim 2009, Hou/Markert/Strube 2014). Hou, Markert und Strube (2014) etwa beschreiben ein regelbasiertes System zur Identifizierung von bridging-Phänomenen. Ihre Algo-

7 Als Anker werden von Schwarz $(1997,2000)$ die sprachlichen Strukturen bezeichnet, die gerade kein direktes, koreferenzielles Antezedens darstellen, sondern die die Grundlage für Inferenzen und indirekte Zusammenhänge zum Referenten der Anapher bilden. Diese Terminologie wird im Zusammenhang mit indirekten Analepsen (vgl. Abschnitt 3.4) übernommen.

8 Nach Lakoff/Johnson (1980) verbinden Metaphern einen bekannten Quellbereich mit einem unbekannten Zielbereich. 
rithmen basieren auf verschiedenen formalen und semantischen Merkmalen wie der Argumentstruktur ${ }^{9}$ der Anapher, der semantischen Konnektivität und semantischen Relationen zwischen NPs, Salienz und Adjazenz (bzw. zeitliche Nähe) (Hou et al. 2014: 2085-2088).

Die skizzierten Erkenntnisse und Vorgehensweisen bei der Resolution oder Vorhersage direkter und indirekter Anaphern sind auch ertragreich für die Analepsenresolution, ${ }^{10}$ also der Identifizierung des Antezedens oder Ankers der Analepse. Der große Unterschied zwischen Analepsen und lexikalischen Anaphern besteht selbstverständlich darin, dass der Bezug der Analepse nicht mittels einer Nominalphrase indiziert wird. Stattdessen muss der Hörer diesen mithilfe seines Diskurs-, Situations- und Weltwissens inferieren. Während es bei lexikalischen Anaphern also explizite sprachliche Anhaltspunkte durch die Nominalphrasen gibt, bietet in Analepsen nur das Verb (weniger explizite) Anhaltspunkte. Im Falle von vorhandenen Leerstellen (also in der Äußerung nicht realisierten Komplementen) kann anhand des Verbs rückgeschlossen werden, mit welcher Art von Ergänzung diese gefüllt werden könnte. So zeigt Plewnia (2003, 2013) anhand dependenzgrammatischer Untersuchungen von Ellipsen, welche (nicht wörtlich, aber kategorial rekonstruierbaren) Ergänzungen von Verben bei diesen nicht realisiert sind (vgl. z. B. Plewnia 2013: 243f.). Bisang (2015) beschreibt, dass eine geringere Explizitheit auf der Formseite mit einer größeren „versteckten Komplexität“ einhergeht, d. h. dass die pragmatischen Inferenzen komplexer werden. Einerseits kann zwar die durch das deutsche Flexionssystem noch relativ explizite Formseite des Verbs bestimmte Antezedenzien restringieren. ${ }^{11}$ Andererseits muss der tatsächliche Zusammenhang mit Antezedenzien im Präkontext durch die Valenzstruktur des Verbs sowie den sequenziellen, pragmatischen und semantischen Kontext inferiert werden. Die

\footnotetext{
9 Nach den Autoren lassen sich bridging-Anaphern u. a. dadurch vorhersagen bzw. Antezedenzien erkennen, dass der Kopf der Antezedens-NP eine Rolle der potenziellen Anapher-NP darstellt (Hou et al. 2014: 2086)

10 Den Begriff ,Analepsenresolution` verwende ich analog zum in der Anaphernforschung und Computerlinguistik gebräuchlichen Begriff ,Anaphernresolution', der die Identifizierung des Antezedens einer Anapher beschreibt.

11 Das Verb der Analepse schränkt die vielfältigen Bezugsmöglichkeiten im Präkontext nicht unbedingt hinsichtlich eines bestimmten Kasus ein (vgl. Abschnitt 3.1 mit Beispielen zu Kasusinkongruenzen). Aber aufgrund der Verbflexion bzw. die Mikrovalenz des Verbs (vgl. Ágel 2000: 220) können z. B. Hörer direkt verstehen, ob eine Analepse adressaten- oder sprecherbezogen ist, wenn die Verbflexion z. B. die 1. P. Sg. oder 2. P. Sg. ist. Ebenso ist bei einem analeptischen Verb in der 3. P. Sg. direkt ersichtlich, dass der Referent eines ausgelassenen Subjekts nicht der Hörer oder Sprecher ist. Die tatsächliche Identifizierung des Antezedens kann bei einem Verb in der 3. P. Sg. in der Analepse aber natürlich nicht durch die Syntax allein geleistet werden.
} 
Prinzipien einer solchen Analepsenresolution sind dabei ähnliche wie die aus der Anaphernforschung bekannten: Auf der Basis syntaktischer und semantischer Analysen kann eine erste Restriktion potenzieller Antezedenzien vorgenommen werden, denn das Verb der Analepse bietet grammatische und semantische Einschränkungen in Bezug auf die größere Menge sprachlicher Strukturen im Präkontext. Eine tatsächliche Identifizierung der Antezedenzien erfolgt in Zusammenhang mit einer semantischen und pragmatischen Analyse weiterer Elemente der Analepse sowie auf der Basis relevanztheoretischer ${ }^{12}$ Überlegungen. Im Falle von indirekten Analepsen, bei denen keine Koreferenz vorherrscht, kann anhand einer semantischen Analyse des Referenten des passenden ,Ankers' und des Bezugs der Analepse (der oft erst auf der Basis des Ankers inferiert werden kann) untersucht werden, in welcher Relation diese zueinander stehen.

\section{Analepsen mit Topik-Drop}

Analeptische Äußerungen können unterschiedliche Arten von Bezügen zu unterschiedlichen Arten von Antezedenzien aufweisen. Eine systematische Untersuchung sowie Darstellung dieser Bezüge ist insofern komplex, als dabei mehrere Ebenen berücksichtigt werden müssen. Zum einen spielen bei der Identifizierung von Antezedenzien die grammatischen Merkmale der Analepse sowie der Antezedenzien eine tragende Rolle. Zum anderen muss jedoch die Interpretation der Analepse im Sinne einer Rekonstruktion der jeweiligen Bezüge auf semantischkonzeptueller Ebene erfolgen.

Auf grammatischer Ebene können Antezedenzien in verschiedener Weise realisiert sein. In den untersuchten Daten sind Antezedenzien Nominalphrasen, Verbalphrasen, Adjektivphrasen, Hauptsätze, Komplement- oder Supplementsätze oder elliptische (Teil-)Sätze.

Für eine systematische Analyse von Analepsen und deren Resolution ist die semantische Ebene $^{13}$ jedoch von größerer Bedeutung, da eine reine Identifizie-

12 Aus den Prinzipien der Relevance Theory (Sperber/Wilson 1986, Wilson/Sperber 2004) lassen sich zwei ausschlaggebende Maximen für die Analepsenresolution gewinnen (vgl. Helmer 2016): 1. Das Antezedens liegt immer so nah wie möglich an der Analepse; 2. Die Identifizierung des Antezedens braucht so wenige Inferenzen wie möglich.

13 Nicht gemeint ist damit z. B. eine Analyse der semantischen Rollen der Antezedenzien. Diese differieren je nach Verb der Analepse und je nach Antezedens so sehr, dass eine systematische Kategorisierung unnötig kompliziert wäre. Im Folgenden wird daher nicht auf semantische Rollen oder valenzgrammatischen Eigenschaften des jeweiligen Verbs eingegangen. Die hier vertretene weite Auffassung von Semantik umfasst hingegen auch das Konzept von Referenz: Bei 
rung der grammatischen Antezedenzien nicht hinreichend für eine Interpretation der Analepsen ist. Bezüge könnten personelle Referenten, Objekte, Prädikationen, abstrakte Konzepte, Ereignisse, Sachverhalte etc. sein. Analepsen können semantisch propositionswertig sein oder auf den Teil einer Proposition (z. B. eine Prädikation, einen Gegenstand etc.) Bezug nehmen. Außerdem sind Bezüge auf einen Sprechakt möglich: In solchen Fällen charakterisieren Sprecher i. d. R. eine vorherige Äußerung als bestimmten Sprechakt oder kommentieren ein einzelnes Lexem in einer bestimmten Weise, z. B. bezüglich seiner Illokution oder seiner Angemessenheit in einem bestimmten Gesprächskontext. Frequent sind zudem indirekte Analepsen, die mit indirekten Anaphern vergleichbar sind (vgl. Schwarz 1997, Schwarz 2000, Consten/Schwarz-Friesel 2007). Bei den sprachlichen Ankern von indirekten Analepsen handelt es sich auf der grammatischen Ebene ebenfalls häufig um Konstituenten, also z. B. eine Nominalphrase oder Verbalphrase. Bei ihnen besteht jedoch keine Koreferenz zwischen dem Referenten des Antezedens bzw. dem Anker im Präkontext und dem, der in der Analepse nicht realisiert ist. Beide Referenten stehen lediglich in einer bestimmten semantischen Relation wie Antonymie, Metonymie usw. Daher bilden indirekte Analepsen ein ganz eigenes Phänomen, das für die Untersuchung der kognitiven Leistungen von Interagierenden besonders interessant ist.

Die im Folgenden analysierten Daten stammen aus einer Kollektion von 541 analeptischen Strukturen mit Topik-Drop. Das Korpus besteht aus 34 Stunden und 48 Minuten informeller Alltagsgespräche (aus FOLK) ${ }^{14}$ und Mediengespräche (GIF), die jeweils als Transkripte und Tondateien, teils als Videoaufzeichnungen vorliegen.

Tabelle 1 gibt einen Überblick über die verschiedenen Typen der 541 untersuchten Analepsen hinsichtlich ihrer Antezedenzien.

der beschriebenen Klassifikation liegt der Schwerpunkt auf der Identifizierung und Beschreibung des Antezedens und seines Referenten und die Identifizierung des Bezugs der Analepse.

14 Alle verwendeten Gespräche aus dem Forschungs- und Lehrkorpus Gesprochenes Deutsch (FOLK) sind online in der Datenbank für gesprochenes Deutsch (dgd.ids-mannheim.de) zu finden. 
Tab. 1: Analepsentypen und ihre Antezedenzien

\begin{tabular}{|c|c|c|c|}
\hline & & & Anzahl \\
\hline $\begin{array}{l}\text { direkte } \\
\text { Analepsen }\end{array}$ & pro & & $\begin{array}{c}225 \\
(41,6 \%) \\
\end{array}$ \\
\hline & & Hauptsatz & $\begin{array}{c}195 \\
(86,7 \% \text { von } 225)\end{array}$ \\
\hline & & Komplementsatz & $\begin{array}{c}13 \\
(5,8 \% \text { von } 225) \\
\end{array}$ \\
\hline & & elliptisch & $\begin{array}{c}17 \\
(7,6 \% \text { von } 225)\end{array}$ \\
\hline & & & $\begin{array}{c}180 \\
(33,3 \%) \\
\end{array}$ \\
\hline & & NP & $\begin{array}{c}42 \\
(19,1 \% \text { von } 180)\end{array}$ \\
\hline & & VP & $\begin{array}{c}24 \\
(10,9 \% \text { von } 180)\end{array}$ \\
\hline & & ADJP & $\begin{array}{c}3 \\
(1,4 \% \text { von } 180)\end{array}$ \\
\hline Sprechakt- $t$ & $\operatorname{sen}$ & & $\begin{array}{c}28 \\
(5,2 \%) \\
\end{array}$ \\
\hline indirekte $\mathrm{A}$ & & & $\begin{array}{c}108 \\
(20,0 \%)\end{array}$ \\
\hline Gesamt & & & 541 \\
\hline
\end{tabular}

Während man durch die bisherige Ellipsen- und Analepsenforschung den Eindruck bekommt, bei Analepsen handle es sich vor allem um Konstituentenanalepsen, zeigt Tabelle 1, dass 41,6\% der Analepsen einen propositionswertigen Bezug haben und sich auf eine gesamte Vorgängeräußerung beziehen, nicht nur auf Konstituenten der Vorgängeräußerung. Von einer rein syntaktische Strukturübernahme kann in diesen Fällen nicht ausgegangen werden. Jedoch ist auch hinsichtlich der Konstituentenanalepsen interessant, ob die Konstituenten der Vorgängeräußerungen syntaktisch in die Analepsen „kopiert“ und dann koordinativ integriert werden oder ob diese Annahme eher nicht zu halten ist.

\subsection{Konstituentenanalepsen}

Analepsen, deren Antezedenzien Nominal-, Verbalphrasen oder Adjektive sind, sind zwar Adjanzenzellipsen, ähneln Koordinationsellipsen aber in Bezug auf strukturelle Aspekte. Die im Folgenden diskutierten Fälle von Analepsen zeigen, dass die Vorstellung einer syntaktischen Strukturübernahme jedoch häufig zu kurz greift.

Ist das Antezedens einer Analepse eine Konstituente wie z. B. eine Nominalphrase, scheint die Annahme naheliegend zu sein, dass es sich um einen Fall von syntaktischer Strukturübernahme handelt, dass also die Analepse direkt an das Antezedens anschließt, das mit denselben grammatischen Eigenschaften etwa im 
phonologischen Speicher repräsentiert ist, ohne konzeptuell repräsentiert werden zu müssen. Dass einige grammatische Antezedenzien denselben Kasus haben wie die an sie anschließenden Analepsen, und dadurch direkt in die Analepsen „passen“, ist unbestreitbar. Jedoch lassen sich Ausnahmen hiervon beobachten, die in Frage stellen, ob eine direkte syntaktische Übernahme bzw. eine aus dem phonologischen Speicher (vgl. Baddeley/Hitch 1974) funktioniert.

Bei Auers (2014) Beispielen ist eine syntaktische Strukturübernahme deshalb möglich, weil es sich bei den verblosen Analepsen i. d. R. um Reparaturkonstruktionen (z. B. Auer 2014: 542) oder um Antworten (z. B. Auer 2014: 557) handelt, die vom jeweiligen Verb der host-Äußerungen regiert werden. Das jeweilige Verb eröffnet dabei die von Auer beschriebene „Strukturlatenz“ und wirkt für spätere Äußerungen gewissermaßen projizierend (Auer 2015). Bei den Analepsen mit Verb in der symbiont-Äußerung wiederum handelt es sich um koordinative Strukturen wie etwa Pro-Drop-Konstruktionen (z. B. Auer 2014: 540), bei denen die Auslassung im Gegensatz zu Topik-Drop-Phänomenen eher sprachstrukturelle Gründe hat. ${ }^{15}$ Wie bereits in Abschnitt 2.1 angedeutet, ist jedoch bei Analepsen, die ein Verb enthalten und die nicht auf koordinativen Strukturen beruhen, eine rein syntaktische Strukturübernahme der host-Äußerung für die symbiont-Äußerung oder eine Übernahme aus dem phonologischen Speicher nicht anzunehmen.

In den untersuchten Daten finden sich auch Fälle, die man mit Strukturlatenzen erklären könnte, nämlich dann, wenn auf grammatischer Ebene Kasuskongruenz des Antezedens und der vom Verb der Analepse regierten (nicht realisierten) Struktur, sowie Koreferenz der entsprechenden Bezüge vorherrscht. Dies ist der Fall in Beispiel 1:

Beispiel 1: Studentisches Alltagsgespräch, FOLK_E_00027_SE_01_T_01, 06:42-06:51

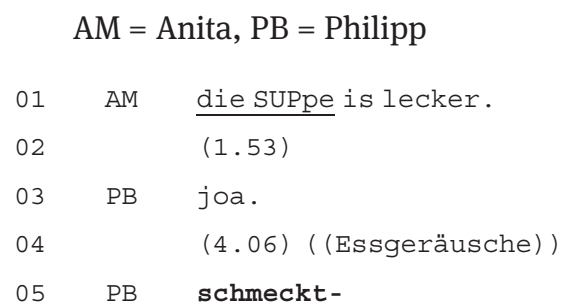

15 Bisang (2015) betont, dass radikaler Pro-Drop nicht einmal mit sprachstrukturellen Gründen, sondern auf Basis pragmatischer Überlegungen erklärt werden muss. Deutsch ist allerdings nicht vergleichbar mit radikalen Pro-Drop-Sprachen oder solchen, in denen Pro-Drop sehr verbreitet ist (wie in einigen romanischen Sprachen). Fälle von Pro-Drop sind in den untersuchten Daten nur dann berücksichtigt, wenn gleichzeitig Topik-Drop auftritt, also das ausgelassene Pronomen als Referent die Person oder das Objekt hat, die/das gleichzeitig Topik ist. 


$\begin{array}{lll}06 & (1.14) \\ 07 & \text { PB } \quad \text { GUT }- \\ 08 & & (5.56)\end{array}$

Die von Anita geäußerte Nominalphrase im Nominativ, „die SUPpe“ (Z. 01), kann in genau dieser Form als host-Struktur für Philipps Analepse ,schmeckt- (1.14) GUT-“ (Z. 05-07) dienen, deren Verb schmecken ein Subjektkomplement regiert, also eine NP im Nominativ. Auf grammatischer Ebene dient daher Anitas NP „die SUPpe“ als Antezedens für den analeptischen Anschluss Philipps. Konzeptuell besteht Koreferenz: „[die suppe] schmeckt- (1.14) GUT-“. Dieses Beispiel kann problemlos wie von Auer (2014) dargestellt werden (vgl. Abb. 4):

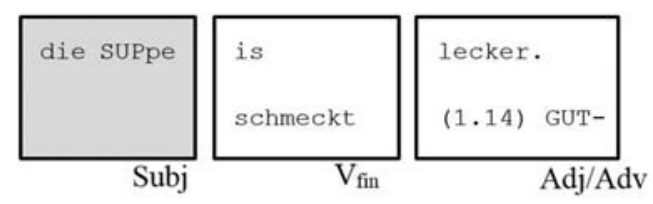

Abb. 4: Antezedens und Analepse in Beispiel 1

In Fällen, in denen der Kasus des Antezedens ein anderer ist als das Verb der Analepse verlangt, könnte man zwar nicht mehr von syntaktischen, aber immerhin von rein phonologischen Strukturübernahmen ausgehen, wie in Beispiel 2, in dem Pascal und seine Freunde einige Vögel im Garten beobachten:

Beispiel 2: Gartengespräch unter Freunden, FOLK_E_00066_SE_01_t_04, 03:28-03:32

$\mathrm{JO}=$ Johnny, PA = Pascal

$\begin{array}{lll}01 & \text { JO } & \text { [wirsind dann demnächst fertig oder] so. } \\ 02 & \text { PA } & \text { [fütter mal VoLLkornbrot; } \\ 03 & & \text { is gSUND für die; }\end{array}$

An seine Aufforderung „fütter mal VOLLkornbrot;“ (Z. 02), schließt Pascal direkt mit der Bewertung „,is gSUND für die;“ (Z. 03) an. In der ersten Äußerung steht die Konstituente „VOLLkornbrot“ im Akkusativ, in der analeptischen Folgeäußerung würde die Kopula eine (hier nicht realisierte) NP im Nominativ regieren. Da der Kasuswechsel selbst bei einer Vollrealisierung hier morphologisch nicht ersichtlich wäre (,[Vollkornbrot] is gSUND für die;“), könnte man annehmen, 
dass die grammatische und valenzsemantische ${ }^{16}$ Inkongruenz aufgrund der Gestaltgleichheit kein Problem darstellt, also die fehlende Struktur in der Analepse vom Sprecher direkt aus der host-Struktur bzw. dem phonologischen Speicher übernommen werden kann.

In den Beispielen 1 und 2 könnte man prinzipiell eine direkte syntaktische/ phonologische Strukturübernahme annehmen, und zwar in 1) aufgrund der Kasuskongruenz und in 2) aufgrund der phonologischen Gestaltgleichheit auch bei Kasuswechsel. Zudem besteht in beiden Fällen Koreferenz zwischen Antezedens und dem nicht durch eine Konstituente realisierten Bezug der Analepse. Bei anderen Belegen gibt es jedoch weder Kasuskongruenz noch phonologische Gestaltgleichheit. So ist in Beispiel 3 der Kasuswechsel auch morphologisch markiert:

\section{Beispiel 3: Gespräche im Fernsehen, GIF_Berlin19_kunst}

HN = Hermann Nitsch

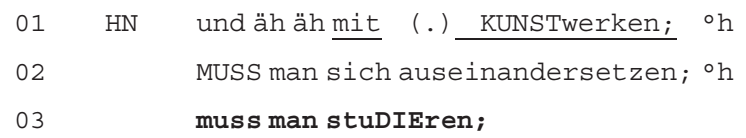

Das Antezedens „(mit) ${ }^{17}$ KUNSTwerken“ (Z. 01) ist eine Nominalphrase im Dativ innerhalb einer Präpositionalphrase. Das Verb der Analepse (Z. 03), studieren, verlangt jedoch ein Akkusativobjekt. Die direkte Kombination „[(mit) (.) KUNSTwerken] muss man stuDIEren;“ wäre agrammatisch („Kunstwerken muss man studieren“) oder würde etwas anderes als das von Nitsch Gemeinte bedeuten (etwa „mithilfe von Kunstwerken muss man studieren“). Hier schlägt demnach eine Strukturübernahme auf der Basis syntaktischer oder phonologischer Gestaltgleichheit fehl. Stattdessen müssen Sprecher und Hörer konzeptuelle „Veränderungen" am Antezedens, besser gesagt an dessen Referent, vornehmen, um die Analepse zu produzieren und zu interpretieren. Analepsen dieser Art entsprechen dem, was Hennig (2010) in historischen Textkorpora als ,aggregative Koordinationsellipsen' untersucht, bei denen realisierte und nicht realisierte Konstituente nicht genau übereinstimmen. Mögliche Numerus- oder Genus-

16 Nicht nur die grammatischen Kasus der von den beiden unterschiedlichen Verben regierten Konstituente wechseln zwischen den Äußerungen, sondern auch die semantischen Rollen der Konstituenten.

17 Ob die Präposition mit Teil des Antezedens ist, kommt auf die Interpretation der Analepse an (s. u.). 
Inkongruenzen ${ }^{18}$ bei der „Kopie“ der Vorgängerstruktur für die analeptische Struktur beschreibt auch Auer (2014: 544). Ebenso erwähnt er das Hinzufügen und Weglassen von Negationspartikeln (Auer 2014: 546f.), die personaldeiktische Adaption der Pronomina und der Verbflexion bei gleichbleibendem Referenten, aber wechselndem Sprecher ( $d u$ vs. ich, ihr vs. wir, vgl. Auer 2014: 552ff.), sowie Chiasmen hinsichtlich der Wortfolge (V1- vs. V2-Stellung, vgl. Auer 2014: 557). Bei seinem Ansatz scheint der Fokus deshalb nicht auf den konzeptuellen „Anpassungen“19 zu liegen, weil diese sich automatisch auf Sprecher- und Hörerseite vollziehen. Eine genaue Analyse solcher Fälle ist jedoch sehr ertragreich für das Verständnis der teils sehr komplexen kognitiven Prozesse, die Sprecher und Hörer vollziehen müssen.

Eine syntaktische oder phonologische Strukturübernahme ist beispielsweise auch dann nicht möglich, wenn eine Analepse an Konstituenten der Vorgängeräußerung prinzipiell anschließen kann, jedoch andere Elemente fehlen, um eine grammatische und sinnhafte Äußerung zu ergeben. Im folgenden Ausschnitt wird in der Analepse eine Präpositionalphrase nicht geäußert, die auch nicht von der host-Äußerung im Sinne einer Strukturlatenz angeboten wird. Andere Konstituenten, hier zwei Nominalphrasen, können dagegen aus der Bezugsäußerung übernommen werden, jedoch nicht in derselben Reihenfolge. Beispiel 4 zeigt diesen komplexen Fall. Vor dem Ausschnitt hatte Darius einen Witz über den Fußballspieler Cacau gemacht und im Zuge dessen Haschisch erwähnt. Der Zusammenhang zwischen den beiden ist Simon nicht klar:

Beispiel 4: Spieleinteraktion zwischen Erwachsenen, FOLK_E_00021_SE_ 01_T_12, 31:05-31:12

$\mathrm{DK}=$ Darius, $\mathrm{SK}=\mathrm{Simon}$

$\begin{array}{lll}01 & \text { SK } & \text { was hat } \text { cacAU mit HAschisch zu tun. } \\ 02 & & (0.27) \\ 03 & \text { DK } & \text { kannst REINtun; }\end{array}$

Simons Frage „was hat cacAU mit HAschisch zu tun.“(Z. 01) beantwortet Darius knapp mit „kannst REINtun;“ (Z. 03). In Simons Frage steht die Nominalphrase „cacAU“ im Nominativ, danach folgt eine Präpositionalphrase mit dem Nomen

18 Mit Inkongruenzen ist hier gemeint, dass das Antezedens einen anderen Numerus, Genus oder Kasus aufweist als die hypothetische Konstituente, die das Verb in der Analepse regieren würde, würde es sich statt um eine Analepse um eine vollständige Äußerung handeln.

19 Auers Fokus auf die Syntax wird auch durch die Bezeichnung ,Strukturlatenzen“ statt etwa ,Konzeptlatenzen' deutlich. 
„HAschisch“ im Dativ. Das Verb reintun (Z. 03) regiert neben einem Subjekt sowohl ein direktes Objekt in Form einer Nominalphrase im Akkusativ als auch eine Präpositionalphrase. Sowohl Subjekt als auch Objekt und adverbiale Bestimmung fehlen in Darius Analepse: Bei dieser sind neben dem Pronomens $d u$ noch zwei weitere Argumente nicht realisiert.

Damit die Gesprächsteilnehmer die Analepse angemessen interpretieren, d. h. idealiter auch das Wortspiel verstehen können, müssen sie teils komplexes Weltwissen abrufen bzw. bestehendes Vorwissen aktivieren und andere kognitive Leistungen erbringen:

1. Sie müssen wissen, dass „Cacau“ ein Name (eines Fußballspielers) ist.

2. Sie müssen erkennen, dass dessen Name homophon mit dem Getränk „Kakao" ist.

3. Sie müssen wissen, dass man Haschisch in zubereiteten Kakao hineingeben und dann den Kakao trinken kann (nicht etwa Haschisch und Kakao mischen und dann rauchen).

4. Sie müssen die einzelnen Konstituenten konzeptuell umstellen, denn eine direkte Übernahme der Nominalphrasen der Bezugsäußerung in ihrer chronologischen Reihenfolge schließt sich aus (vgl. Inferenz 3 oben): In der hostÄußerung wird zuerst „Cacau“, dann „Haschisch“ genannt, die Analepse erfordert genau die umgekehrte Reihenfolge.

5. Damit zusammenhängend müssen sie konzeptuell spezifizieren, in was man Haschisch reintun kann. Auf rein grammatischer Ebene kann die Analepse nicht an die Antezedenzien anschließen, da in der Bezugsäußerung keine passende Präposition geäußert wurde. Auch wenn das Konzept eines Behältnisses (bzw. dessen Inhalts) durch das Adverb rein aktualisiert wird, muss doch konzeptuell spezifiziert werden, dass das, wo etwas reingetan werden kann, das Getränk Kakao ist.

Abbildung 5 illustriert die genannten Punkte anhand der konzeptuellen Rekonstruktion auf der Basis der Antezedenzien. 


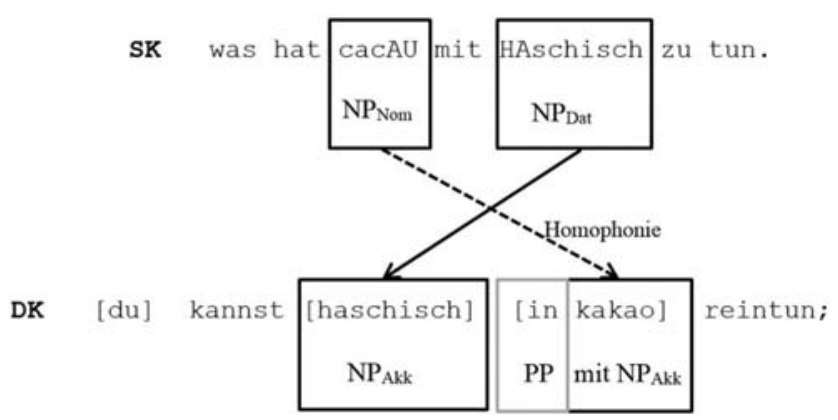

Abb. 5: Bezugsäußerung mit Antezedenzien und Analepse mit nicht realisiertem Bezug in Beispiel 5

Alle genannten Punkte legen nahe, dass statt einer direkten syntaktischen Strukturübernahme oder einer Übernahme aus dem phonologischen Speicher das konzeptuelle Verständnis der einzelnen Konstituenten und ihrer Relationen zentral ist. Auch die im Folgenden diskutierten semantisch komplexeren Analepsen (vgl. Abschnitte 3.2 bis 3.4) sowie Experimente der Gedächtnispsychologie (z. B. Atkinson/Shiffrin 1968) deuten darauf hin, dass nicht (nur) das lexikalische Material im Kurz- oder Langzeitgedächtnis wortgetreu gespeichert wird, sondern von der lexikalischen Ebene abstrahierte Informationen memoriert und erinnert werden. Bei einer Latenz zwischen Antezedens und Analepse, die länger als 2 Sekunden ${ }^{20}$ beträgt, sind selbst einfache Konstituentenanalepsen mit Kasuskongruenz, Gestaltgleichheit und Koreferenz wahrscheinlich besser durch „konzeptuelle Latenzen“ als durch syntaktische Strukturlatenzen oder phonologische Gestaltgleicheit zu erklären.

\subsection{Analepsen mit propositionswertigem Bezug}

Bisher wurden Analepsen mit Bezug auf einzelne Konstituenten untersucht, um an diesen Beispielen zu zeigen, wieso eine rein syntaktische Strukturübernahme nicht plausibel ist. In der zugrundeliegenden Kollektion sind Analepsen, die einfache Konstituenten auslassen, gegenüber solchen mit komplexeren Bezügen

20 Baddeley und Hitch (1974) schätzen den phonologischen Speicher auf der Basis von Experimenten auf etwa 1-2 Sekunden. Eine längere Speicherung ist nur möglich, wenn die phonologische Information in der phonologischen Schleife landet, also mit einer „inneren Stimme“ ständig aktualisiert wird. Dies ist angesichts des laufenden Gesprächsflusses eine eher nicht haltbare Annahme. 
jedoch ohnehin in der Unterzahl. Wie Tabelle 1 in Abschnitt 3 zeigt, sind im Vergleich zu (koreferenziellen) Konstituentenanalepsen solche Analepsen frequenter, deren Antezedenzien an einen propositionswertigen Sachverhalt anschließen (oder einen anderen Bezug haben, vgl. auch Abschnitt 3.3 und 3.4). Auch hier ist eine direkte Strukturübernahme aus dem phonologischen Speicher nur in den seltenen Fällen (5,8 \% aller propositionswertigen Bezüge) denkbar, in denen die host-Äußerung einen Komplement- oder Supplementsatz darstellt, an den wiederum die Analepse direkt anschließt, i. d. R. in Form eines syntaktischen Parallelismus zur Bezugsäußerung (vgl. Mitkov 1999). Gewöhnlich schließen Analepsen mit propositionswertigem Bezug jedoch an Deklarativsätze mit Verbzweitstellung an; ein direkter syntaktischer Anschluss der Analepse wäre agrammatisch. Auch in diesen Fällen scheint daher die konzeptuelle Ebene von zentraler Bedeutung für die Interagierenden zu sein, $d . h$. die mentale Repräsentation der Proposition.

Einen typischen Beleg zeigt Beispiel 5, in dem sich Norbert und Elena über Norberts Bruder unterhalten, der kürzlich mit seiner Freundin zusammengezogen ist:

Beispiel 5: Pärchengespräch, FOLK_E_00039_SE_01_T_01, 15:20-15:29

$\mathrm{NO}=$ Norbert, EL = Elena,

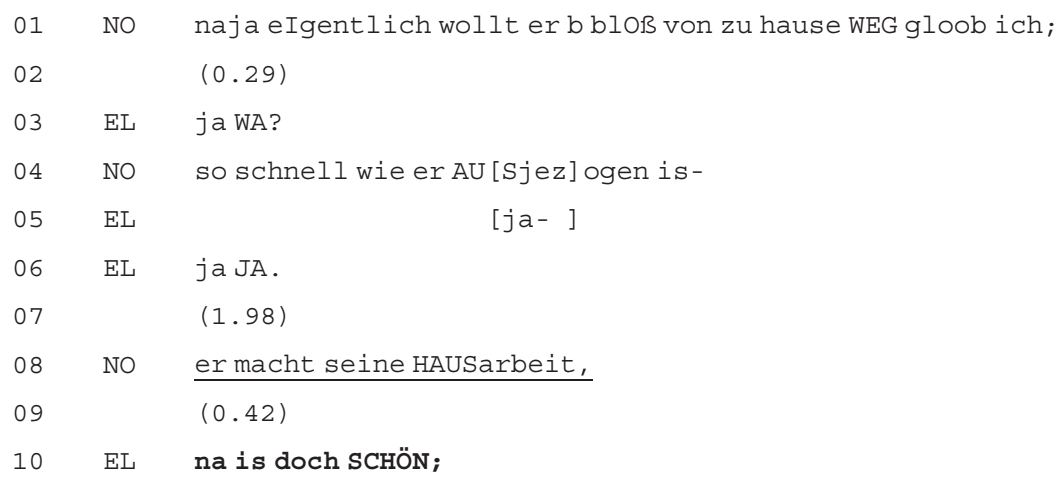

Die Kopula sein in Elenas analeptischer Bewertung „na is doch SCHÖN;“ (Z. 10) kann ein Subjekt, eine Infinitivkonstruktion oder einen Komplementsatz regieren. Aus pragmatischen Gründen kann ausgeschlossen werden, dass Elena sich auf die Äußerung „elgentlich wollt er b bloß von zu hause WEG“ (Z. 01) bezieht, und ebenso, dass sie die Hausarbeit an sich als schön bewertet. Ihre Bewertung bezieht sich vielmehr auf den adjazenten Turn von Norbert „er macht seine HAUSarbeit,“ (Z. 08), ist also der second pair part eines Adjazenzpaars (vgl. Schegloff 2007: 13ff.). Norberts Äußerung ist propositionswertig: „Norberts Bruder macht seine Hausarbeit“. Die Analepse schließt aber nicht direkt syntaktisch an den Haupsatz an, 
stattdessen rückt der Schwerpunkt auf den konzeptuellen Sachverhalt. Paraphrasiert werden kann dies nur mittels eines Nebensatzes, der jedoch nicht geäußert wird: „[dass er seine hausarbeit macht] is doch SCHÖN;“.

Analepsen dieser Art sind sehr frequent, sie machen 41,6 \% aller untersuchten analeptischen Äußerungen aus. Das Antezedens ist dabei nicht immer in einer einzigen (Teil-)Äußerung zu finden, sondern kann sich auch über mehrere (Teil-) Äußerungen erstrecken, d. h. in mehreren Äußerungen stehen jeweils Elemente, die zusammen eine Proposition ergeben. Zudem kann der Skopus von Analepsen selbst bei propositionswertigen Antezedenzien weniger oder mehr als das Antezedens selbst umfassen. Dies betrifft insbesondere Modalpartikeln, Negationen und Modalverben, die etwa für die Bezugsäußerung konstitutiv sind, für die Interpretation der Analepse jedoch konzeptuell ausgeblendet werden müssen oder umgekehrt für diese konzeptuell „hinzugefügt“ werden müssen. Diese Fälle stützen ebenfalls die These, dass die Propositionen bzw. Teile eines potenziellen propositionalen Bezugs $^{21}$ konzeptuell repräsentiert werden.

\subsection{Analepsen mit Sprechaktbezug}

In der bisherigen Forschung noch nicht untersucht wurden Analepsen, die eine Äußerung als einen bestimmten Sprechakt, i. S. der Illokution einer sprachlichen Handlung, charakterisieren, z. B. als Frage, als Vorwurf oder als Bitte. In diesen Fällen kann das Antezedens eine Konstituente oder propositionswertig sein, jedoch unterscheiden sich die Fälle von denen in den Abschnitten 3.1 und 3.2 insofern, als Sprecher mit der Analepse die Bezugsäußerung als eine spezifische Sprechhandlung charakterisieren. Sprecher nehmen in diesen Fällen also auf die Illokution einer vorherigen Äußerung Bezug.

Beispiel 6 stammt aus einer Interaktion, in der einige Freunde ein Fußballmanager-Spiel spielen, in dem sie Fußballspieler kaufen und diese zu (fiktiven) Mannschaften zusammensetzen. In dem Ausschnitt wird dem Mitspieler Simon nach einer Frage Naivität oder mangelndes Nachdenken unterstellt, woraufhin er seine Frage inhaltlich abschwächt:

21 Die Bezüge über mehrere Äußerungen hinweg können nur potenziell propositional sein, da erst durch die Analepse selbst klar wird, ob das Topik die Proposition als Ganzes ist oder nur Teile dieser, also z. B. ein personeller Referenten in Form einer NP als Antezedens. 


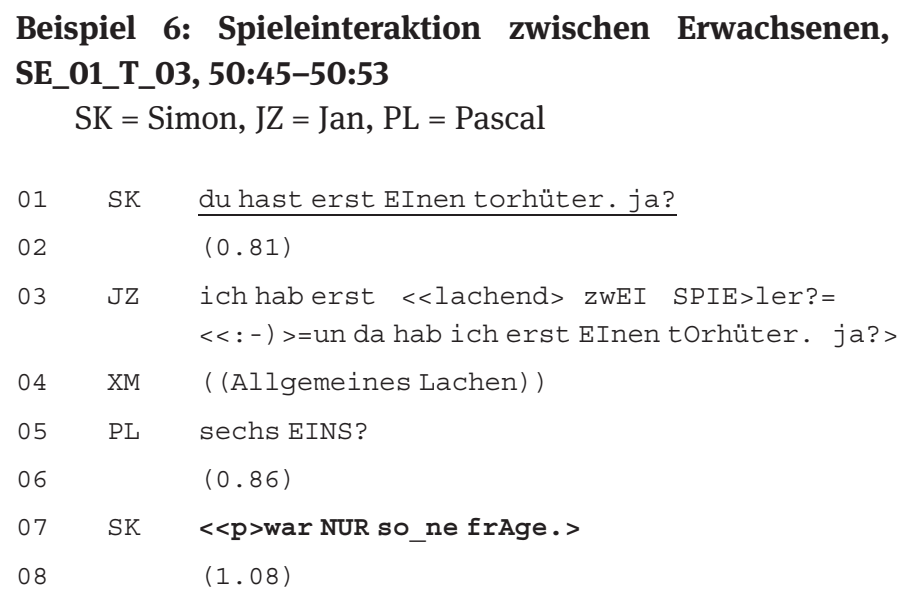

Jan fasst Simons Frage, ob er erst einen Torhüter ersteigert habe, offenbar als Spott auf; er rechtfertigt, bei zwei bisher ersteigerten Spielern sei es normal erst einen Torhüter zu besitzen. Simon interpretiert diese Rechtfertigung wiederum als Vorwurf mangelnder Relevanz oder Überlegung, also Illegitimität seiner Frage, was wiederum eine Rechtfertigung seinerseits projiziert. In seiner analeptischen Äußerung „war NUR so_ne frAge.“ (Z. 07) schwächt er seine zuvor gestellte Frage mit der Partikel „NUR“ und dem modalisierten indefiniten Artikel „so_ne“'22 ab, nimmt ihr damit die Gewichtigkeit, die Jans Rechtfertigung ihr zuschreibt, und charakterisiert sie als einfache, wertungsfreie Informationsfrage.

Die Bezugsäußerung der Analepse liegt dabei in Zeile 01; jedoch ist der Bezug selbst nicht der Äußerungsinhalt, also die Proposition, sondern die Illokution der Äußerung. Das Antezedens könnte zwar konzeptuell propositional eingefügt werden, dies würde aber den Charakter der Illokution nicht treffen, denn der springende Punkt ist, welche sprachliche Handlung die Äußerung in Zeile 01 vollzieht: Statt „[ob du erst einen torhüter hast,] war NUR so_ne frAge.“ ist gemeint: „[meine Äußerung] war NUR so_ne frAge.“. Durch seine Analepse versucht Simon, einen face-threatening-act (vgl. Brown/Levinson 1987) abzuwenden, denn Jan impliziert mit seiner Äußerung, Simons anfängliche Frage sei illegitim oder unpassend. Simons analeptische Äußerung ist hier als eine Form von third-position self repair (vgl. Schegloff 1992) zu verstehen, mit dem der selbst geäußerte Turn retrospektiv re-interpretiert und modalisiert wird.

Wie das Beispiel verdeutlicht, sind auch Analepsen auf der Sprechaktebene keine Analepsen, die hinreichend aufgrund von Strukturübernahmen oder der

22 Vgl. zu so ein/e Hole/Klumpp (2000) und von Heusinger (2011). 
Übernahme einer Äußerung aus dem phonologischen Speicher erklärt werden kann. Die Ebene der Interpretation wechselt hier von der Sachverhaltsebene auf die Sprechaktebene; ${ }^{23}$ Simon bezieht sich nicht auf seine zuvor geäußerte Proposition, sondern auf deren Illokution (die im Übrigen in Z. 01 nicht dieselbe sein muss wie Z. 07 vermuten lässt - Simon könnte seine Bezugsäußerung tatsächlich ursprünglich als spöttischen Scherz, implizite Kritik an Jans Expertise o. Ä. gemeint haben).

\subsection{Indirekte Analepsen}

Am ergiebigsten sind ,indirekte Analepsen“ (analog zu ,indirekten Anaphern', vgl. Abschnitt 2.2), um 1. spezifische kognitive Leistungen der Interagierenden bei ihrer Produktion und Interpretation zu demonstrieren, und um 2. zu zeigen, dass ein direkt syntaktischer Anschluss von Analepsen, selbst wenn augenscheinlich möglich, nicht zu einer hinreichenden Interpretation der Äußerung führen kann. Indirekte Analepsen sind mit $20 \%$ relativ häufig und erscheinen i. d. R. zuerst wie Konstituentenanalepsen (vgl. Abschnit 3.1). Bei indirekten Analepsen besteht allerdings keine Koreferenz zwischen dem nicht realisierten Topik der Analepse und dem Referenten des ,Ankers“ (Schwarz 1997: 449). Stattdessen besteht zwischen den beiden Referenten eine spezifische semantische Relation. Ein Beispiel dafür ist Beleg 4 in Abschnitt 3.1, in dem der Referent des Ankers „cacAU“ nicht mit dem Referenten der Analepse „Kakao“ übereinstimmt - stattdessen stehen diese beiden Ausdrücke in einem homophonen ${ }^{24}$ Verhältnis. Indirekte Analepsen hängen also nur indirekt mit Elementen des Präkontextes zusammen; für deren Produktion und Interpretation sind komplexere Inferenzen nötig als bei direkten Analepsen. Abbildung 7 verdeutlicht den Unterschied zwischen direkten und indirekten Analepsen.

$23 \mathrm{Zu}$ der Unterscheidung zwischen Sachverhalts- und Sprechaktebene vgl. auch Sweetser (1990).

24 Als Spezialfall von Homonymie wird Homophonie hier als eine semantische Relation zwischen zwei Ausdrücken betrachtet. 

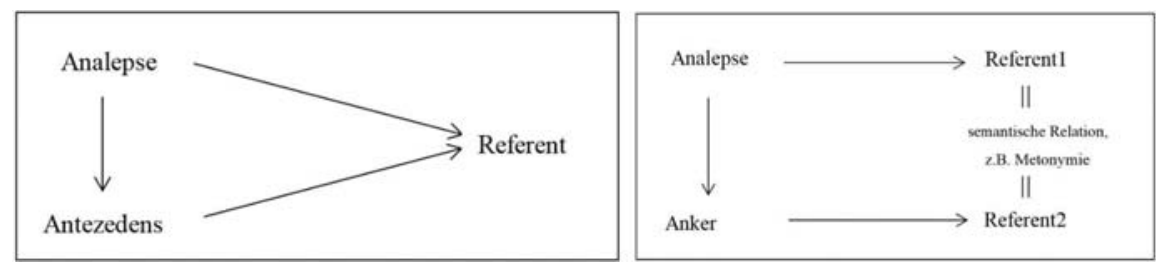

Abb. 6: Die Verhältnisse zwischen Analepse, Antezedens/Anker und Referenten bei direkten und indirekten Analepsen

Auch einige Interaktionslinguisten stellen fest, dass nicht alle Analepsen durch direkte Strukturübernahmen auf den Präkontext erklärbar sind. Wie bereits beschrieben, weist Auer (2014) etwa auf konzeptuelle Anpassungen (ich vs. $d u$, wir vs. ihr und die damit einhergehenden Verbflexionen) hin. Lindström/Karlsson (2005: 113) erwähnen sogar mögliche implikatorische Bezüge von Analepsen. Eine systematische Analyse, welche solcher konzeptuellen Veränderungen und implikatorischen Bezüge üblich sind, ist bisher jedoch nicht erfolgt. Im Folgenden werden zwei Beispiele für indirekte Analepsen diskutiert, bei denen der Referent der Analepse keine Koreferenz mit dem Referent des sprachlichen Ankers der Analepse aufweist. Stattdessen stehen die Referenten miteinander in einer semantischen Relation.

\subsubsection{Homonymie/Polysemie}

Neben Homophonie (s. o.) gibt es in den untersuchten Daten einige Fälle von Homonymie und Polysemie zwischen zwei Bezügen. Im Falle von Polysemie hat ein Lexem mehrere Bedeutungen, Homonymie beruht auf der phonologisch identischen Repräsentation verschiedener Lexeme. Die Ambiguität des Ankers kann dabei z. B. für einen Witz genutzt werden (vgl. Attardo (1994)). ${ }^{25}$ In Beispiel 7 macht sich Nico die Ambiguität für ein Wortspiel zunutze, das darauf basiert, dass der Bezug des Ankers und der der Analepse homonym sind:

25 Attardo (1994: 115) stellt dazu fest: „As all puns, paradigmatic puns involve two senses of a linguistic expression (or string). What sets these apart from other types of puns is that only one of the two strings is actually present in the text (the uttered string), and the second has to be retrieved by the hearer from his/her storage of homonymic or patronymic strings“. 


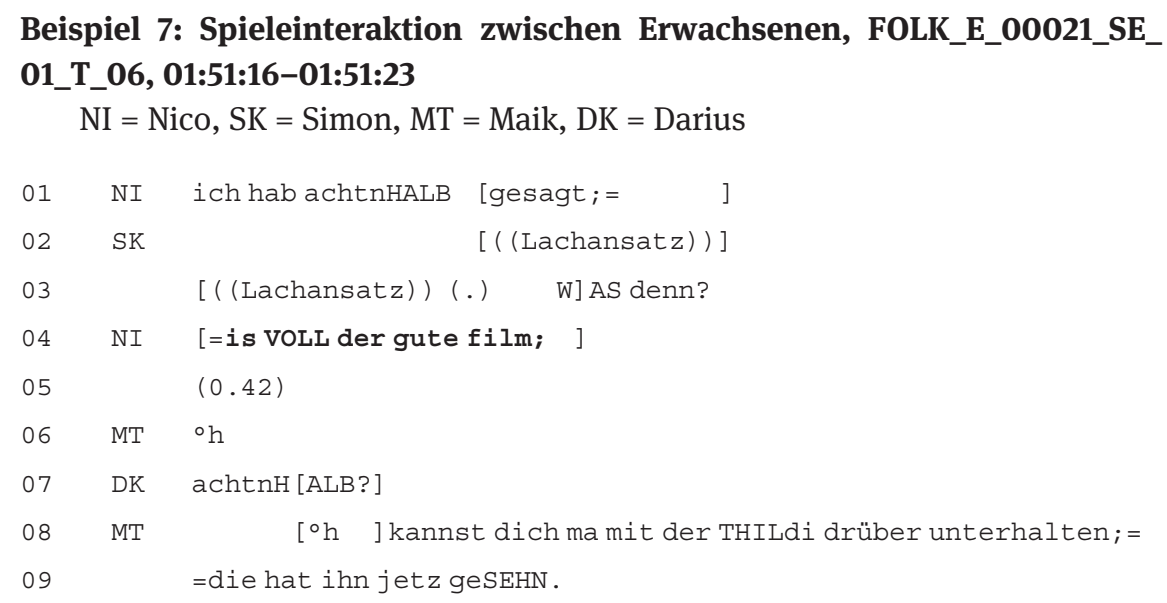

Während des Fußballmanager-Spiels bietet Nico für einen Fußballspieler 8,5 Millionen Euro. Er wiederholt den Betrag ,achtnHALB“ in einem Selbstzitat (Z. 01), wobei dieses Zahlwort sich in der Äußerung auf den Euro-Geldbetrag bezieht (also: 8,5 Millionen Euro). Daraufhin schließt Nico unmittelbar mit einer weiteren Teiläußerung an und sagt über „achtnHALB“ aus: „is VOLL der gute film;“ (Z. 04). Mit dieser Analepse bezieht er sich jedoch nicht mehr auf einen Geldbetrag, sondern auf einen Kinofilm des italienischen Regisseurs Federico Fellini, der den Titel „8 1/2“ trägt. Das Zahlwort „achtnHALB“ der vorherigen Äußerung ist damit zwar auf der grammatischen Ebene das Antezedens bzw. der Anker der Analepse und kann im Sinne einer rein phonologischen Übernahme als direkte Ergänzung dafür dienen. Jedoch handelt es sich nicht um eine direkte Analepse, sondern um eine indirekte. Obwohl selbstverständlich das Lexem achteinhalb selbst in beiden Fällen ein Zahlwort ist, also Polysemie vorliegt, kann man in gewissem Sinne von pragmatischer Homonymie sprechen, denn ,achtnHALB“ wechselt von seinem Status eines Geldbetrags zu einem Eigennamen bzw. Filmtitel: Die Prädikativkonstruktion $X$ ist NP erfordert hier ein Subjekt, nicht ein Adjektiv bzw. eine Zahl. Nico nutzt die Homonymie von „achtnHALB“ für einen witzig gemeinten Kommentar, der auf dem typischen Muster einfacher (Wort-)Witze beruht und der von mindestens einem Mitspieler auch verstanden wird, da diesem und seiner Partnerin der Film bekannt ist (Z. 08-09).

Wie Beispiel 7 und ebenso Beispiel 4 zeigen, funktionieren die Witze gerade deshalb, weil die Analepsen nicht auf Strukturübernahmen oder phonologischer Gestaltgleichheit beruhen. Die Analepsen können zwar rein grammatisch durch den Anker vervollständigt werden und die Wortspiele an sich würden natürlich auch funktionieren, wenn die Sprecher das jeweilige Antezedens der Vorgängeräußerung wiederholen würden, also keine Analepsen äußern würden. Dennoch 
liegt der Witz gerade darin, dass die Sprecher dies nicht tun und die Antezedenzien (,cacau“ und „,achnthalb“) mit deren jeweiligen, in den Vorgängeräußerung gültigen Bezügen stehen lassen, also die Hörer selbst die Inferenz ziehen müssen, dass die zuerst gültigen Bezüge nicht die der Analepse sein können.

\subsubsection{Metonymie}

Häufiger als homonyme Relationen finden sich Fälle, in denen der Referent der Analepse in einer metonymischen Beziehung zum Referenten des Ankers steht. Die metonymische Relation kann dabei zeitlich oder prozessual sein oder eine pars-pro-toto-Relation, bei dem einer der Referenten einen Teil des anderen Referenten (Teil-für-Ganzes) darstellt (vgl. hierzu Markert 1999). Eine metonymische Relation zwischen den Referenten zeigt Beispiel 8 aus einem Gespräch eines Paares, das eine Woche zuvor geheiratet hatte. Die beiden, Elena (EL) und Norbert (NO) unterhalten sich über ihren Hochzeitstag:

Beispiel 8: Pärchengespräch, FOLK_E_00039_SE_01_T_01, 13:42-14:07 $\mathrm{EL}=$ Elena, $\mathrm{NO}=$ Norbert

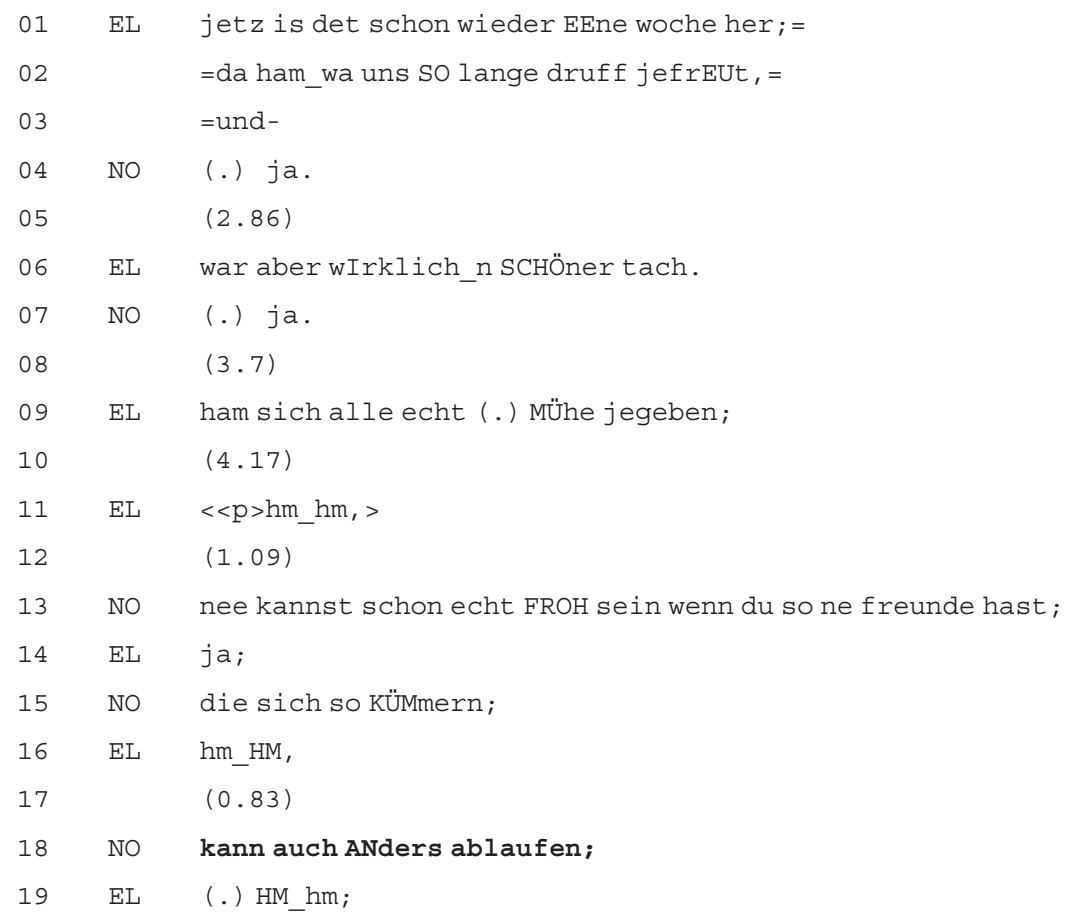


Durch ihre Äußerung in Zeile 01 etabliert Elena den gemeinsamen Hochzeitstag als Diskurstopik. ${ }^{26}$ Nachdem sie diesen durch „war aber wIrklich_n SCHÖner tach.“ (Z. 06) positiv bewertet, stellen sie und ihr Mann interaktiv fest, dass der Erfolg u. a. auf die gemeinsamen Freunde zurückzuführen ist, die bei der Organisation mitgeholfen haben (Z. 09-16). Daraufhin stellt Norbert mit einer Analepse fest: „kann auch ANders ablaufen;“ (Z. 18). Das prozessuale Verb ablaufen der Analepse regiert eine Subjektergänzung, semantisch ist diese stark restringiert: Was abläuft, kann nur eine Zeitspanne oder ein direkt mit einer Zeitspanne verbundenes Konzept sein. Einige Nominalphrasen wie „so ne freunde“ (Z. 13) und „MÜhe“ (Z. 09) können daher als Antezedenzien ausgeschlossen werden. Das zeitlich nächste passende Antezedens ist das Subjekt der Äußerung in Zeile 06 „n schöner tach“, mit dem Elena lexikalisch ihre Anapher „det“ in Zeile 01 näher spezifiziert. Beide nehmen Bezug auf das Diskurstopik - den Hochzeitstag.

Der eigene Hochzeitstag, auf den Elena sich mit „det“ (Z. 01) und „tach“ (Z. 06) bezieht, hat hier eine token-Referenz und meint den speziellen, vergangenen Hochzeitstag der beiden, der positiv verlaufen ist. Norberts Analepse jedoch bezieht sich nicht auf den eigenen Hochzeitstag, sondern auf Hochzeitstage allgemein im Sinne einer type-Referenz. Es besteht eine zweifache pars-pro-totoRelation: Das Diskurstopik bzw. „tach“ (pars) steht für den positiven Hochzeitstag Elenas und Norberts. Was anders ablaufen kann, sind aber Hochzeitstage generell (toto). In diesem Sinne liegt das semantische Verhältnis der Hyponymie bzw. Hyperonymie vor, bei dem die Bedeutungen der untergeordneten Wörter die der übergeordneten Wörter im Sinne einer hierarchischen Strukturierung enthalten (Schwarz/Chur 1996: 57).

Zum anderen ist natürlich der Tag als zeitliche Einheit nur ein Teil des ganzen Komplexes, den eine Hochzeit ausmacht. Norbert und Elena beziehen sich explizit darauf, dass sich ihre Freunde gekümmert haben (Z. 15), was auch die Vorbereitungen, also die Organisation im Vorfeld einschließt, inklusive der Erstellung einer vor dem Ausschnitt erwähnten Hochzeitszeitung u. Ä. „tach“ steht auch unter dieser Sichtweise als pars, jedoch zusätzlich für eine größere zeitliche Spanne und für einen größeren Komplex von Geschehnissen (toto).

Dass der Begriff des Hochzeitstags metonymisch auch für damit zusammenhängende Prozesse generell verwendet werden kann, und dass er generisch für Hochzeitstage interpretiert werden kann, ist natürlich auch unabhängig von der Interpretation der Analepse der Fall. Auch sind metonymische Relationen und konzeptuelle Prozeduren bei deren Interpretation nicht spezifisch für Analepsen.

26 Der Hochzeitstag war zuvor nicht explizit Diskurstopik, sondern die Hochzeitszeitung, die von Freunden der beiden für die Feier angefertigt worden war. 
Fest steht dennoch, dass die Verwendung (und Interpretation) indirekter Analepsen von Sprechern hier und in anderen Fällen genutzt wird, um mittels einer ökonomischen Sprechweise zu interagieren, Witze zu machen (wie etwa in Beispiel 4 und 7) oder Interpretationsspielräume offen zu lassen. Die Erklärung durch eine rein syntaktische Strukturübernahme ohne die Berücksichtigung kognitiver Prozesse ist in diesen Fällen nicht möglich.

\section{Zusammenfassung und Diskussion}

Die diskutierten Beispiele zeigen, dass die Produktion und Interpretation von Analepsen häufig komplexer sind als oft angenommen wird. In fast allen zitierten Interaktionsausschnitten ist es nicht möglich, dass die Antezedenzien oder Anker Strukturlatenzen für eine folgende Analepse bieten oder direkt aus dem phonologischen Speicher aktualisiert werden können. Selbst wenn es ein syntaktisch „passendes“ Antezedens ist, reicht dessen Identifizierung v. a. bei Inkongruenzen hinsichtlich Numerus, Kasus, Genus/Sexus, und noch vielmehr bei indirekten Analepsen nicht aus, um zu erklären, wie Sprecher diese Analepsen überhaupt bilden und wie Hörer sie verstehen können. Konzeptuelle Prozesse bei der Produktion und Interpretation zumindest der Analepsen, die selbst ein Verb enthalten, sind vielmehr die Regel als die Ausnahme.

Zusammenfassend deuten die folgenden Punkte darauf hin:

- Bisweilen gibt es Kasus- und Numerusinkongruenzen zwischen den Konstituenten, die Antezedenzien von Analepsen sind, und den Konstituenten, die von den Verben der Analepsen regiert werden, dabei jedoch nicht realisiert werden. Während man noch von einer Übernahme aus dem phonologischen Speicher ausgehen kann, wenn die Inkongruenzen sich nicht auf der morphologischen Ebene zeigen (vgl. Bsp. 2 in Abschnitt 3.1), ist dies nicht möglich, wenn es im Falle einer Vollrealisierung der Analepsen morphologische Veränderungen der Konstituenten gäbe (vgl. Bsp. 3 in Abschnitt 3.1).

- Relativ häufig (in den untersuchten Daten etwa 20 \%) sind Analepsen, die wie Konstituentenanalepsen erscheinen, aber keine direkten Analepsen, sondern indirekte Analepsen sind. In diesen Fällen besteht keine Koreferenz zwischen den Referenten der Anker und den Referenten der Analepsen (bzw. ihrer nicht realisierten Konstituenten). Stattdessen stehen die Referenten in bestimmten semantischen Relationen zueinander. Am häufigsten sind dies metonymische Relationen, in den untersuchten Daten sind aber auch Homonymie/Polysemie, Antonymie und Homophonie zwischen den Referenten zu finden. Sowohl für die Produktion von Analepsen auf Sprecherseite als auch für deren Interpretation auf Hörerseite sind dabei teils komplexe Inferenzen nötig. 
- Generell sind Analepse und Antezedens oder Anker oft keineswegs in adjazenten Äußerungen realisiert, sondern es kann eine bisweilen so große Latenz zwischen beiden bestehen, dass allein aufgrund syntaktischer Überlegungen nicht zu erklären ist, wieso ausgerechnet die letztlich identifizierte Konstituente als Antezedens fungiert und nicht etwa eine andere, die ebenfalls syntaktisch passen könnte und sogar zeitnäher an der Analepse liegt. Eine Übernahme aus dem phonologischen Speicher ist bei einer Latenz von mehr als 1-2 Sekunden ohnehin nicht möglich.

Noch frequenter sind Fälle, in denen die Antezedenzien gar keine einfachen Konstituenten sind, sondern andere sprachliche Strukturen:

- Sehr häufig (41,6 \%) haben Analepsen einen propositionwertigen Bezug. Eine direkte Strukturübernahme wäre in fast allen diesen Fällen agrammatisch, da der Bezug nur in seltenen Fällen ein Komplement- oder Supplementsatz ist, an den eine Analepse mit V1-Stellung direkt anschließen kann. Fast immer handelt es sich beim Bezug um Hauptsätze. Auch in diesen Fällen ist also die mentale Repräsentation der Propositionen entscheidend, nicht etwa eine syntaktische Passung.

- Das Antezedens kann selbst eine Ellipse und dabei semantisch propositionswertig sein, z. B. eine adjektivische Bewertung wie „sehr schön“ mit Bezug auf ein von Sprecher und Hörer wahrgenommenes Objekt. Eine Analepse (wie etwa „find ich auch“) kann sich wiederum auf diese elliptisch geäußerte Bewertung beziehen. Zur Produktion bzw. Interpretation der Analepse muss bei Sprecher bzw. Hörer nicht nur das Antezedens konzeptuell repräsentiert sein, sondern auch das bewertete Objekt. Ebenfalls kann der Skopus von Analepsen auch bei propositionswertigen Antezedenzien weniger oder mehr als das Antezedens selbst umfassen. Dies betrifft insbesondere Modalpartikeln, Negationen und Modalverben, die etwa für die Bezugsäußerung konstitutiv sind, für die Interpretation der Analepse jedoch konzeptuell ausgeblendet werden oder umgekehrt für diese konzeptuell „hinzugefügt““ werden müssen (Helmer 2016: 123ff.).

- Einige Analepsen haben einen Sprechaktbezug, d. h. sie beziehen sich auf eine Sprechhandlung und charakterisieren diese retrospektiv in einer bestimmten Weise (z. B. als Witz oder als Frage). Dabei wäre ein direkter syntaktischer Anschluss i.d. R. agrammatisch. Ein syntaktischer Fokus würde außerdem nicht die teils komplexen kognitiven Vorgänge bei ihrer Produktion und Interpretation erklären, in diesem Fall das Wechseln von der Sachverhaltsebene auf die Sprechaktebene.

Sowohl für Konstituentenanalepsen, die nicht auf syntaktischer Strukturübernahme basieren können, als auch für Analepsen, deren Antezedenzien keine 
Konstituenten sind, muss angenommen werden, dass statt der syntaktischen Ebene die konzeptuelle Repräsentation der Referenten oder komplexeren Bezüge die entscheidende Rolle bei der Produktion und Interpretation von Analepsen spielt. Die Sinnhaftigkeit von analeptischen Äußerungen muss in Verbindung mit dem Präkontext aktiv hergestellt werden. Dabei wirken bei der Identifizierung des Antezedens bzw. Ankers und damit auch bei der Interpretation der Analepse die grammatischen Merkmale der Analepse in einem ersten Schritt zwar restringierend. Jedoch reichen die grammatischen Restriktionen nicht aus, um eine Analepse hinreichend $\mathrm{zu}$ interpretieren und $\mathrm{zu}$ inferieren, was genau das nicht realisierte Topik ist. Vielmehr sind es semantische Eigenschaften des Verbs und weiterer Elemente innerhalb der Analepse, sowie pragmatische Aspekte, auf deren Basis innerhalb der eingeschränkten Bezugsmöglichkeiten ein passendes Antezedens identifiziert und daraufhin die Analepse interpretiert werden kann.

Statt der syntaktischen Ebene in Form von Strukturlatenzen und Strukturübernahme sind also konzeptuelle Repräsentationen und kognitive Prozesse auf Sprecher- und Hörerseite ausschlaggebend. Analepsen können, sofern sie selbst ein Verb enthalten, deutlich komplexere Bezüge haben als in der Ellipsenforschung bisher angenommen wurde. Der in der interaktionslinguistischen Ellipsenforschung bisher bestehende (syntaktische) Fokus auf Konstituentenanalepsen sollte demnach um kognitive Ansätze erweitert werden (wie sie in der Psycholinguistik z. T. auch schon üblich sind, vgl. etwa Rickheit/Strohner 2008, Rickheit/Sichelschmidt 2013). Auch Bisang (2015) betont, dass die pragmatische Komponente im Sinne von Inferenzprozessen stärkere Beachtung finden muss, wo Explizitheit auf der Formseite gering ist.

Durch eine Kombination des sequenzanalytischen Ansatzes mit (mikro- und makro-)valenzgrammatischen, semantischen und pragmatischen Analysen kann aufgezeigt werden, unter welchen Bedingungen sprachliche Ökonomie seitens der Sprecher überhaupt möglich ist und wann hingegen keine Disambiguierung der semantisch unterspezifizierten analeptischen Äußerungen für den Hörer durchführbar ist. Dabei können auch Aussagen darüber getroffen werden, wie Analepsen geplant und verstanden werden (müssen), sodass es im weiteren Diskursverlauf nicht zu Missverständnissen kommt. Zwar ist die Tatsache, dass keine Missverständnisse angezeigt werden, noch kein Beweis für die Gültigkeit der eigenen Hypothesen über die kognitiven Prozesse von Sprechern und Hörern. Dagegen kann aber die Analyse von abweichenden Fällen (deviant case analysis, vgl. Hutchby/Wooffitt 2008: 91f.), in denen Hörer ihr Nichtverstehen anzeigen, die Grenzen der Analepseninterpretation im Falle eines zu aufwändigen oder gar unmöglichen Verarbeitungsprozesses aufzeigen (vgl. Helmer 2016). Wenn, wie üblich, keine Verständnisschwierigkeiten zu beobachten sind oder Sprecher sogar durch sichtbare Aufzeigehandlungen (displays, vgl. Schegloff/Sacks 1973, Scheg- 
loff 1997, Hutchby/Wooffitt 2008: 13) zeigen, dass sie sich an Kognitionen bzw. kognitiven Zuschreibungen orientieren, kann mit einer großen Wahrscheinlichkeit davon ausgegangen werden, dass bestimmte kognitive Prozesse bei Sprecher und Hörer erfolgen: Sie sind aufmerksam, sie erinnern sich an das Gesagte (oder Teile davon) und sie verstehen das Gesagte. Speziell Hörer müssen bei einer hinreichenden Analepseninterpretation außerdem zwischen potenziellen Antezedenzien selegieren und im Falle von indirekten Analepsen die tatsächlichen Referenten der analeptischen Äußerungen auf der Basis sprachlicher Anker inferieren.

Konversationsanalyse und Interaktionale Linguistik positionieren sich in Bezug auf die Untersuchung von Mentalem traditionell eher agnostisch bis sogar antimentalistisch. Neuere Untersuchungen (z. B. Imo 2011, Deppermann 2012) hingegen betonen, dass Annahmen über kognitive Prozesse von Interagierenden unvermeidbar sind, und sogar anhand konversationsanalytischer und interaktionslinguistischer Methoden nachweisbar sind. Diese Annahme wird durch die vorliegende Untersuchung gestützt, indem sie aufdeckt, dass Annahmen über spezifische kognitive Prozesse bei der Produktion und Interpretation der grammatisch und semantisch unterspezifizierten analeptischen Strukturen unabdingbar sind.

\section{Literatur}

Ágel, Vilmos (2000): Valenztheorie. Tübingen: Narr.

Atkinson, Richard C./Shiffrin, Richard M. (1968): Human Memory: A Proposed System and Its Control Processes. In: Kenneth W. Spence \& Janet T. Spence (Hg.), The psychology of learning and motivation. Vol. 2. New York: Academic Press, S. 89-195.

Attardo, Salvatore (1994): Linguistic theories of humor. Berlin/New York: de Gruyter. (Humor research 1).

Auer, Peter (1993): Zur Verbspitzenstellung im gesprochenen Deutsch. In: Deutsche Sprache 21 (3), S. 193-222.

Auer, Peter (2000): On Line-Syntax - Oder: was es bedeuten könnte, die Zeitlichkeit der mündlichen Syntax ernst zu nehmen. In: Sprache und Literatur 31(1), S. 43-56.

Auer, Peter (2006): Increments and more. Anmerkungen zur augenblicklichen Diskussion über die Erweiterbarkeit von Turnkonstruktionseinheiten. In: Arnulf Deppermann/Reinhard Fiehler/Thomas Spranz-Fogasy (Hg.), Grammatik und Interaktion. Radolfzell: Verlag für Gesprächsforschung, S. 279-294.

Auer, Peter (2007): Syntax als Prozess. In: Heiko Hausendorf (Hg.), Gespräch als Prozess: Linguistische Aspekte der Zeitlichkeit verbaler Interaktion. Tübingen: Narr, S. 95-124. (Studien zur deutschen Sprache 37).

Auer, Peter (2014): Syntactic structures and their symbiotic guests. Notes on analepsis from the perspective of on-line syntax. In: Pragmatics 24(3), S. 533-560. 
Auer, Peter (2015): The temporality of language in interaction. Projection and latency. In: Arnulf Deppermann/Susanne Günthner (Hg.), Temporality in interaction. Amsterdam/Philadelphia: Benjamins, S. 27-56.

Baddeley, Alan D. \& Hitch, Graham J. (1974): Working memory. In: Gordon H. Bower (Hg.), The psychology of learning and motivation: Advances in research and theory. Vol. 8. New York: Academic Press, S. 47-90.

Behr, Irmtraud/Quintin, Hervé (1996): Verblose Sätze im Deutschen. Zur syntaktischen und semantischen Einbindung verbloser Konstruktionen in Textstrukturen. Tübingen, Stauffenburg. (Eurogermanistik 4).

Bisang, Walter (2015): Hidden complexity - the neglected side of complexity and its implications. In: Linguistic Vanguard. Online verfügbar: http://www.degruyter.com/view/j/lingvan.ahead-of-print/lingvan-2014-1014/lingvan-2014-1014.xml

Bücker, Jörg (2012): Sprachhandeln und Sprachwissen: Grammatische Konstruktionen im Spannungsfeld von Interaktion und Kognition. Berlin/Boston: de Gruyter. (Sprache und Wissen 11).

Busler, Christine/Schlobinski, Peter (1997): „Was er (schon) (...) konstruieren kann - das sieht er (oft auch) als Ellipse an.“ Über ,Ellipsen', syntaktische Formate und Wissensstrukturen. In: Peter Schlobinski (Hg.), Syntax des gesprochenen Deutsch. Opladen: Westdeutscher Verlag, S. 93-115.

Carnie, Andrew (2013): Syntax: A generative introduction. 3. Aufl. Malden, MA: Wiley-Blackwell. (Introducing linguistics 4).

Clark, Herbert H. (1975): Bridging. In Roger C. Schank/Bonnie L. Nash-Webber (Hg.), Theoretical issues in natural language processing. New York: Association for Computing Machinery, S. 169-174.

Consten, Manfred/Marx, Konstanze (2006): Komplex-Anaphern - Rezeption und textuelle Funktion. In: Hardarik Blühdorn/Eva Breindl/Ulrich H. Waßner (Hg.), Text - Verstehen. Grammatik und darüber hinaus. Berlin/New York: de Gruyter, S. 375-379. (Jahrbuch des Instituts für Deutsche Sprache 2005).

Consten, Manfred/Schwarz-Friesel, Monika (2007): Anapher. In: Ludger Hoffmann (Hg.), Handbuch der deutschen Wortarten. Berlin/New York: de Gruyter, S. 265-292. (de Gruyter Lexikon).

Deppermann, Arnulf (2006): Deontische Infinitivkonstruktionen: Syntax, Semantik, Pragmatik und interaktionale Verwendung. In: Susanne Günthner/Wolfgang Imo (Hg.), Konstruktionen in der Interaktion. Berlin/New York: de Gryuter, S. 239-262. (Linguistik - Impulse \& Tendenzen 20).

Deppermann, Arnulf (2007): Grammatik und Semantik aus gesprächsanalytischer Sicht. Berlin/ New York: de Gruyter. (Linguistik - Impulse \& Tendenzen 14).

Deppermann, Arnulf (2012): How does 'cognition' matter to the analysis of talk-in-interaction? In: Language Sciences 34(6), S. 746-767.

Du Bois, John W. (2014): Towards a dialogic syntax. In: Cognitive Linguistics 25(3), S. 359-410.

Engel, Ulrich (2009): Deutsche Grammatik. Neubearbeitung. 2. Aufl. München: ludicium.

Eroms, Hans-Werner (2000): Syntax der deutschen Sprache. Berlin/New York: de Gruyter. (de Gruyter Studienbuch).

Günthner, Susanne (2006): Grammatische Analysen der kommunikativen Praxis - „Dichte Konstruktionen“ in der Interaktion. In: Arnulf Deppermann/Reinhard Fiehler/Thomas SpranzFogasy (Hg.), Grammatik und Interaktion - Untersuchungen zum Zusammenhang von grammatischen Strukturen und Gesprächsprozessen. Radolfzell: Verlag für Gesprächsforschung, S. 95-122. 
Harweg, Roland (1968): Pronomina und Textkonstitution. München: Fink. (Poetica: Beihefte zu Poetica 2).

Hawkins, John A. (1978): Definiteness and indefiniteness. London: Croom Helm. (Croom Helm Linguistics Series).

Helbig, Gerhard/Buscha, Joachim (1999): Deutsche Grammatik: Ein Handbuch für den Ausländerunterricht. 19. Aufl. Berlin: Langenscheidt.

Helmer, Henrike (2016): Analepsen in der Interaktion. Semantische und sequenzielle Eigenschaften von Topik-Drop im gesprochenen Deutsch. Heidelberg: Winter. (OraLingua 13)

Hennig, Mathilde (2010): Aggregative Koordinationsellipsen im Neuhochdeutschen. In: Ziegler, Arne (Hg.): Historische Textgrammatik und Historische Syntax des Deutschen. Traditionen, Innovationen, Perspektiven. Unter Mitarbeit von Christian Braun. Berlin/New York: de Gruyter, S. $937-963$

Hennig, Mathilde (2013) (Hg.): Die Ellipse. Neue Perspektiven auf ein altes Phänomen. Berlin/ Boston: de Gruyter. (Linguistik - Impulse \& Tendenzen 52).

Hoffmann, Ludger (1997a): Analepse. In: Gisela Zifonun/Ludger Hoffmann/Bruno Strecker et al., Grammatik der deutschen Sprache. (Bd. 1) Berlin/New York: de Gruyter, S. 569-583. (Schriften des Instituts für Deutsche Sprache 7).

Hoffmann, Ludger (1999): Ellipse und Analepse. In: Angelika Redder/Jochen Rehbein (Hg.), Grammatik und mentale Prozesse. Tübingen: Stauffenburg, S. 69-90. (Stauffenburg Linguistik 7).

Hole, Daniel/Klumpp, Gerson (2000): Definite type and indefinite token: The article son in colloquial German. In: Linguistische Berichte 182, S. 231-244.

Hou, Yufang/Markert, Katja/Strube, Michael (2014): A rule-based system for unrestricted bridging resolution: recognizing bridging anaphora and finding links to antecedents. In: Proceedings of the 2014 Conference on Empirical Methods in Natural Language Processing (EMNLP 2014), Doha, Qatar, S. 2082-2093.

Hutchby, Ian/Wooffitt, Robin (2008): Conversation Analysis. 2. Aufl. Cambridge: Polity.

Imo, Wolfgang (2011): Cognitions are not observable - but their consequences are: Mögliche Aposiopese-Konstruktionen in der gesprochenen Alltagssprache. In: Gesprächsforschung Online-Zeitschrift zur verbalen Interaktion 12, S. 265-300. (http://www.gespraechsforschung-ozs.de/heft2011/ga-imo.pdf)

Imo, Wolfgang (2013): Ellipsen und Verstehen in der Interaktion. In: Mathilde Hennig (Hg.), Die Ellipse. Neue Perspektiven auf ein altes Phänomen. Berlin/Boston: de Gruyter, S. 281-319. (Linguistik - Impulse \& Tendenzen 52).

Imo, Wolfgang (2016): Ellipsen, Inkremente und Fragmente aus interaktionaler Perspektive. In: Francois Marillier/Elodie Vargas (Hg.), Fragmentarische Äußerungen. Tübingen: Stauffenburg, S. 217-245.

Kindt, Walther (1985): Grammatische Prinzipien sogenannter Ellipsen und ein neues Syntaxmodell. In: Reinhard Meyer-Hermann/Hannes Rieser (Hg.), Ellipsen und fragmentarische Ausdrücke. Tübingen: Niemeyer, S. 161-290.

Kindt, Walther (2013): Theoretische und methodische Grundlagen der Ellipsenmodellierung. In: Mathilde Hennig (Hg.), Die Ellipse. Neue Perspektiven auf ein altes Phänomen. Berlin/ Boston: de Gruyter, S. 39-106. (Linguistik - Impulse \& Tendenzen 52).

Klein, Wolfgang (1993): Ellipse. In: Joachim Jacobs/Arnim von Stechow/Wolfgang Sternefeld/ Theo Vennemann (Hg.), Syntax. Vol. 1. Ein internationales Handbuch der zeitgenössischen Forschung. Berlin/New York: de Gruyter, S. 763-799. (Handbücher zur Sprach- und Kommunikationswissenschaft 9). 
Krifka, Manfred/Musan, Renate (2012): Information Structure: Overview and linguistic issues. In: Manfred Krifka/Renate Musan (Hg.), The Expression of Information Structure. Berlin/ Boston: de Gruyter, S. 1-443.

Kubczak, Helmut (2012): Anaphorische Konstruktion und antezedensloser Rückverweis. In: Viktoria Ilse/Zoltan Szendi (Hg.), Jahrbuch der ungarischen Germanistik 2011. Budapest/ Bonn: Gondolat Kiadói Kör/DAAD, S. 221-247. (Reihe Germanistik).

Lakoff/Johnson (1980): Metaphors we live by. Chicago: University of Chicago Press.

Lambrecht, Knud (1994): Information structure and sentence form. Topic, focus and the mental representations of discourse referents. Cambridge: Cambridge University Press.

Lindström, Jan/Karlsson, Susanna (2005): Verb-first constructions as a syntactic and functional resource in (spoken) Swedish. In: Nordic Journal of Linguistics 28(1), S. 97-131.

Markert, Katja (1999): Metonymien - eine computerlinguistische Analyse. Sankt Augustin: Infix.

Markert, Katja/Hahn, Udo (2002): Metonymies in discourse. In: Artificial Intelligence 135(1-2), S. $145-198$.

Markert, Katja/Nissim, Malvina (2005): Comparing knowledge sources for nominal anaphora resolution. In: Computational Linguistics 31(3), S. 367-402.

Markert, Katja/Nissim, Malvina (2009): Data and models for metonymy resolution. In: Language Resources and Evaluation 43(2), S. 123-138.

Mitkov, Rusland (1999): Anaphora resolution: the state of the art. Working paper, (Based on the COLING'98/ACL'98 tutorial on anaphora resolution). University of Wolverhampton, Wolverhampton.(http://clg.wlv.ac.uk/papers/mitkov-99a.pdf)

Musan, Renate (2002): Informationsstrukturelle Dimensionen im Deutschen. Zur Variation der Wortstellung im Mittelfeld. In: Zeitschrift für Germanistische Linguistik 30(2), S. 198-221.

Ortner, Hanspeter (1987): Die Ellipse: ein Problem der Sprachtheorie und Grammatikbeschreibung. Tübingen: Niemeyer. (Reihe Germanistische Linguistik 80).

Plewnia, Albrecht (2003): Sätze, denen nichts fehlt. Eine dependenzgrammatische Untersuchung elliptischer Konstruktionen. Am Beispiel des mitteldeutschen Dialekts des Ermlands. Hildesheim/Zürich/New York: Georg Olms. (Germanistische Linguistik Monographien 11).

Plewnia, Albrecht (2013): Möglichkeiten und Wahrscheinlichkeiten. Was man in der Dependenzgrammatik mit syntaktischen Leerstellen tun kann. In: Mathilde Hennig (Hg.), Die Ellipse. Neue Perspektiven auf ein altes Phänomen. Berlin/Boston: de Gruyter, S. 227-251. (Linguistik - Impulse \& Tendenzen 52).

Rath, Rainer (1979): Kommunikationspraxis: Analysen zur Textbildung und Textgliederung im gesprochenen Deutsch. Göppingen: Vandenhoeck und Ruprecht. (Kleine VandenhoeckReihe 1452).

Reinhart, Tanya (1981): Pragmatics and linguistics: an analysis of sentence topics. In: Philosophica 27(1), S. 53-94.

Rickheit, Gert/Sichelschmidt, Lorenz (2013): Verstehen von Ellipsen - ein holistischer Ansatz. In: Mathilde Hennig (Hg.), Die Ellipse. Neue Perspektiven auf ein altes Phänomen. Berlin/ Boston: de Gruyter, S. 159-182. (Linguistik - Impulse \& Tendenzen 52).

Rickheit, Gert/Strohner, Hans (2008): Inferenzen. In: Gert Rickheit/Theo Herrmann/Werner Deutsch (Hg.), Psycholinguistik. Berlin: de Gruyter, S. 566-577. (Handbücher zur Sprachund Kommunikationswissenschaft 24).

Schegloff, Emanuel A. (1997): Whose text? Whose context? In: Discourse \& Society 8(2), S. 165187.

Schegloff, Emanuel A./Sacks, Harvey (1973): Opening up closings. In: Semiotica 8(4), S. 289327. 
Schegloff, Emanuel A. (2007). Sequence organization in interaction: A Primer in Conversation Analysis 1. Cambridge: Cambridge University Press.

Schwarz, Monika (1997): Anaphern und ihre diversen Antezedenten: Koreferenz und Konsorten. In: Christa Dürscheid/Karl-Heinz Ramers/Monika Schwarz (Hg.), Sprache im Fokus. Festschrift für Heinz Vater zum 65. Geburtstag. Tübingen: Niemeyer, S. 445-455.

Schwarz, Monika (2000): Indirekte Anaphern in Texten. Studien zur domänengebundenen Kohärenz und Referenz im Deutschen. Tübingen: Niemeyer. (Linguistische Arbeiten 413).

Selting, Margret (1997): Sogenannte ,Ellipsen` als interaktiv relevante Konstruktionen? Ein neuer Versuch über die Reichweite und Grenzen des Ellipsenbegriffs für die Analyse gesprochener Sprache in der konversationellen Interaktion. In: Peter Schlobinski (Hg.), Syntax des gesprochenen Deutsch. Opladen: Westdeutscher Verlag, S. 117-155.

Sperber, Dan/Wilson, Deirdre (1986): Relevance. Communication and cognition. Oxford: Blackwell.

Sweetser, Eve (1990): From etymology to pragmatics. Metaphorical and cultural aspects of semantic structure. (= Cambridge Studies in Linguistics 54). Cambridge: Cambridge University Press.

Vieira, Renata/Poesio, Massimo (2000): An empirically based system for processing definite descriptions. In: Computational Linguistics 26(4), S. 525-579.

von Heusinger, Klaus (2011): Specificity, referentiality and discourse prominence: German indefinite demonstratives. In: Reich, Ingo/Horch, Eva/Pauly, Dennis (Hg.): Sinn \& Bedeutung. 15th Proceedings of the 2010 Annual Conference of the Gesellschaft für Semantik. 9.11. September 2010, Saarbrücken: Saarland University Press, S. 9-30.

Wilson, Deirdre/Sperber, Dan (2004): Relevance Theory. In: Laurence R. Horn/Gregory Ward (Hg.), The Handbook of Pragmatics. Oxford: Blackwell, S. 607-632. (Blackwell handbooks in linguistics 16).

Zifonun, Gisela/Hoffmann, Ludger/Strecker, Bruno/Ballweg, Joachim/Brauße, Ursula/Breindl, Eva/Engel, Ulrich/Frosch, Helmut/Hoberg, Ursula/Vorderwülbecke, Klaus (1997): Grammatik der deutschen Sprache (Bd. 1-3). Berlin/New York: de Gruyter. (Schriften des Instituts für Deutsche Sprache 7).

\section{Anhang}

Verwendete Transkriptionskonventionen nach GAT 2 (Selting et al. 2009)

Sequenzielle Struktur/Verlaufsstruktur

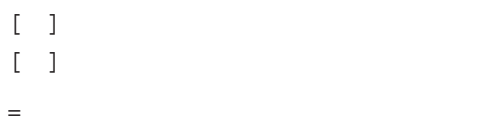

Überlappungen und Simultansprechen

schneller, unmittelbarer Anschluss neuer Sprecherbeiträge oder Segmente (latching)

Ein- und Ausatmen

$\circ \mathrm{h} / \mathrm{h} \circ$

Ein- bzw. Ausatmen von ca. 0.2-0.5 Sek. Dauer

$\circ$ hh / hho

$\circ$ hhh / hhho

Ein- bzw. Ausatmen von ca. 0.5-0.8 Sek. Dauer

Ein- bzw. Ausatmen von ca. 0.8-1.0 Sek. Dauer 
Pausen

\begin{tabular}{l}
$()$. \\
$(-)$ \\
$(0.5)$ \\
Sonstige segmentale Konventionen \\
\hline
\end{tabular}

und_äh
äh öh äm
$:$
$:$ :
$::$ :
?

Akzentuierung

akZENT

akzEnt

ak! ZENT!
Mikropause, geschätzt, bis ca. 0.2 Sek. Dauer

kurze geschätzte Pause von ca. 0.2-0.5 Sek. Dauer

gemessene Pausen von ca. 0.5 Dauer

Verschleifungen innerhalb von Einheiten

Verzögerungssignale, sog. ,gefüllte Pausen`

Dehnung, Längung, um ca. 0.2-0.5 Sek.

Dehnung, Längung, um ca. 0.5-0.8 Sek.

Dehnung, Längung, um ca. 0.8-1.0 Sek.

Abbruch durch Glottalverschluss

Fokusakzent

Nebenakzent

extra starker Akzent

Tonhöhenbewegung am Ende von Intonationsphrasen

\begin{tabular}{|c|c|}
\hline$?$ & hoch steigend \\
\hline , & mittel steigend \\
\hline- & gleichbleibend \\
\hline ; & mittel fallend \\
\hline . & tief fallend \\
\hline \multicolumn{2}{|l|}{ Verändertes Tonhöhenregister } \\
\hline$<<h>>$ & hohes Tonhöhenregister \\
\hline \multicolumn{2}{|l|}{ Lachen und Weinen } \\
\hline haha hehe hihi & silbisches Lachen \\
\hline$(($ lacht $)) \quad(($ weint $))$ & Beschreibung des Lachens \\
\hline$<<\Theta>\mathrm{SOO}>$ & „smile voice“ \\
\hline \multicolumn{2}{|l|}{ Rezeptionssignale } \\
\hline $\mathrm{hm}$ ja nein nee & einsilbige Signale \\
\hline hm_hm ja_a nei_ein nee_e & zweisilbige Signale \\
\hline \multicolumn{2}{|l|}{ Sonstige Konventionen } \\
\hline ((hustet)) & $\begin{array}{l}\text { para- und außersprachliche Handlungen und Ereig- } \\
\text { nisse }\end{array}$ \\
\hline$(x x x),(x x x x x x)$ & ein bzw. zwei unverständliche Silben \\
\hline (solche) & vermuteter Wortlaut \\
\hline (also/alo) (solche/welche) & mögliche Alternativen \\
\hline$[\ldots]$ & Auslassung im Transkript \\
\hline
\end{tabular}


Lautstärke- und Sprechgeschwindigkeitsveränderungen, mit Extensionen

\begin{tabular}{ll}
\hline$<\mathrm{f}>>$ & forte, laut \\
$<<\mathrm{p}>>$ & piano, leise \\
$<<\mathrm{all}>>$ & allegro, schnell \\
$<<\mathrm{dim}>>$ & diminuendo, leiser werdend \\
$<<\mathrm{acc}>>$ & accelerando, schneller werdend
\end{tabular}

